\title{
Downregulation of TrkB Expression and Signaling by Valproic Acid and Other Histone Deacetylase Inhibitors ${ }^{\left[{ }^{[}\right.}$
}

\author{
DSimona Dedoni, Luisa Marras, (1) Maria C. Olianas, Angela Ingianni, and (D) Pierluigi Onali \\ Laboratory of Cellular and Molecular Pharmacology, Section of Neurosciences, Department of Biomedical Sciences (S.D., \\ M.C.O., P.O.) and Section of Microbiology, Department of Biomedical Sciences (L.M., A.I.), University of Cagliari, Cagliari, Italy \\ Received March 15, 2019; accepted June 14, 2019
}

\begin{abstract}
Valproic acid (VPA) has been shown to regulate the levels of brain-derived neurotrophic factor (BDNF), but it is not known whether this drug can affect the neuronal responses to BDNF. In the present study, we show that in retinoic acid-differentiated SH-SY5Y human neuroblastoma cells, prolonged exposure to VPA reduces the expression of the BDNF receptor TrkB at the protein and $\mathrm{mRNA}$ levels and inhibits the intracellular signaling, neurotrophic activity, and prosurvival function of BDNF. VPA downregulates TrkB and curtails BDNF-induced signaling also in differentiated Kelly and LAN-1 neuroblastoma cells and primary mouse cortical neurons. The VPA effect is mimicked by several histone deacetylase (HDAC) inhibitors, including the class I HDAC inhibitors entinostat and romidepsin. Conversely, the class II HDAC inhibitor MC1568, the HDAC6 inhibitor tubacin, the HDAC8 inhibitor PCl-34051, and the VPA derivative valpromide have no effect. In neuroblastoma cells and primary neurons both VPA and entinostat increase the cellular levels of the transcription factor RUNX3, which negatively regulates TrkB gene expression. Treatment with RUNX3 siRNA attenuates VPA-induced RUNX3 elevation and TrkB downregulation. VPA, entinostat, HDAC1 depletion by siRNA, and 3-deazaneplanocin A (DZNep), an inhibitor of the polycomb repressor complex 2 (PRC2), decrease the PRC2 core component EZH2, a RUNX3 suppressor. Like
\end{abstract}

VPA, HDAC1 depletion and DZNep increase RUNX3 and decrease TrkB expression. These results indicate that VPA downregulates TrkB through epigenetic mechanisms involving the EZH2/RUNX3 axis and provide evidence that this effect implicates relevant consequences with regard to BDNF efficacy in stimulating intracellular signaling and functional responses.

\section{SIGNIFICANCE STATEMENT}

The tropomyosin-related kinase receptor $\mathrm{B}$ (TrkB) mediates the stimulatory effects of brain-derived neurotrophic factor (BDNF) on neuronal growth, differentiation, and survival and is highly expressed in aggressive neuroblastoma and other tumors. Here we show that exposure to valproic acid (VPA) downregulates TrkB expression and functional activity in retinoic acid-differentiated human neuroblastoma cell lines and primary mouse cortical neurons. The effects of VPA are mimicked by other histone deacetylase (HDAC) inhibitors and HDAC1 knockdown and appear to be mediated by an epigenetic mechanism involving the upregulation of RUNX3, a suppressor of TrkB gene expression. TrkB downregulation may have relevance for the use of VPA as a potential therapeutic agent in neuroblastoma and other pathologies characterized by an excessive BDNF/TrkB signaling.

\section{Introduction}

The short-chain branched fatty acid valproic acid (VPA) is commonly used in the clinic as an anticonvulsant for the treatment of various forms of epilepsy, as a mood stabilizer for the management of bipolar mood disorder, and as an analgesic for migraine headaches (Loscher, 2002). Moreover, in clinical trials VPA has been investigated as a potential anticancer agent for both hematologic and solid tumors (Chateauvieux et al., 2010). This wide array of pharmacological properties likely stems from the ability of VPA to display multiple

This study was supported by Sardegna Ricerche, Progetto IBERNAT-NBLP.O.R. Sardegna 2014-2020, CUP F21B17000730005.

https://doi.org/10.1124/jpet.119.258129.

S This article has supplemental material available at jpet.aspetjournals.org. mechanisms of action, including inhibition of voltage-gated sodium and calcium channels, potentiation of GABAergic transmission, attenuation of glutamatergic function, and alteration of the activity of distinct intracellular signaling mediators (Loscher, 2002). Moreover, VPA at clinically relevant concentrations has been demonstrated to act as an effective inhibitor of histone deacetylases (HDACs) (Gottlicher et al., 2001; Phiel et al., 2001), which play a critical role in the epigenetic regulation of gene transcription (Thiagalingam et al., 2003; Bolden et al., 2006). Among the four distinct classes of mammalian HDACs so far identified, VPA preferentially inhibits HDACs belonging to class I and IIa (Bolden et al., 2006).

A number of preclinical studies have shown that VPA can exert neuroprotective effects against a variety of brain insults, suggesting a therapeutic potential in different neurodegenerative

ABBREVIATIONS: Akt, protein kinase B; BDNF, brain-derived neurotrophic factor; DZNep, 3-deazaneplanocin A; ERK1/2, extracellular signalregulated kinases 1 and 2; EZH2, enhancer of zeste homolog 2; FCS, fetal calf serum; GAPDH, glyceraldehyde 3-phosphate dehydrogenase; GSK$3 \beta$, glycogen synthase kinase-3 $\beta$; HDAC, histone deacetylase; MAP2, microtubule-associated protein 2; PARP, poly (ADP-ribose) polymerase; PI3K, phosphatidylinositol 3-kinase; PLC $\gamma$, phospholipase $\mathrm{C} \gamma$; PMSF, phenylmethylsulphonyl fluoride; PRC2, polycomb repressive complex 2; qPCR, quantitative polymerase chain reaction; RA, all-trans retinoic acid; siRNA, small interfering RNA; TrkB-FL, TrkB full length isoform; TrkB-T, TrkB truncated isoforms; VPA, valproic acid. 
diseases (Nalivaeva et al., 2009; Chiu et al., 2013). In this context, particular relevance has gained the observation that VPA can upregulate the expression of brain-derived neurotrophic factor (BDNF), a neurotrophin that promotes activity-dependent neuronal growth, differentiation, and survival (McAllister et al., 1999; Park and Poo, 2013). In vitro studies using primary cultures of rat cortical neurons have shown that BDNF expression induced by either VPA or the HDAC inhibitors sodium butyrate and trichostatin A was associated with increased histone acetylation and mimicked by silencing of HDAC1 gene (Yasuda et al., 2009). A microarray analysis of gene expression in cultured rat cortical neurons revealed that BDNF was among the 726 genes upregulated by VPA exposure (Fukuchi et al., 2009). The ability of VPA to target $\mathrm{BDNF}$ transcription may have pharmacological relevance, since alterations of BDNF signaling have been associated with several neuropathological conditions, including epilepsy, affective disorders. and pain, which are clinically treated with VPA (Autry and Monteggia, 2012).

In contrast to the large amount of information available on the regulation of BDNF by VPA, relatively little is known on the effects of this drug on TrkB, the receptor that mediates the biologic actions of BDNF (Chao, 2003; Huang and Reichardt, 2003). Upon binding of neurotrophin homodimers, TrkB, which belongs to the tyrosine kinase receptor family, dimerizes, autophosphorylates, and activates three main signaling pathways, including phosphatidylinositol 3-kinase (PI3K)/ protein kinase B (Akt), phospholipase $\mathrm{C} \gamma(\mathrm{PLC} \gamma)$, and extracellular signal-regulated kinases 1 and 2 (ERK1/2). A number of studies have shown that an efficient activation of each of these pathways is ultimately responsible for the correct control of neuronal growth, differentiation, and survival by the BDNF/TrkB receptor complex (Chao, 2003; Huang and Reichardt, 2003).

The transcriptional regulation of Trk receptor expression is known to be under control of different transcription factors (Lei and Parada, 2007). In developing sensory neurons and human neuroblastoma cells, RUNX3, a runt domain transcription factor, has been shown to selectively repress TrkB expression (Kramer et al., 2006; Inoue et al., 2007). In different cell types the RUNX3 gene is epigenetically silenced by the histone methyltransferase EZH2, a core component of the polycomb repressive complex 2 (PRC2), which has been found to interact and cooperate with class I HDACs (van der Vlag and Otte, 1999; Varambally et al., 2002; Fujii et al., 2008). HDAC inhibitors have been shown to cause depletion of PRC2 proteins and the consequent RUNX3 de-repression (Fiskus et al., 2006; Wang et al., 2012).

To investigate the effect of VPA on TrkB expression and functional activity, we employed human neuroblastoma cell lines, in which TrkB is induced by treatment with retinoic acid (Kaplan et al., 1993), and primary cultures of mouse cortical neurons, which endogenously express the receptor. We also examined whether in these cells VPA could alter the expression of the EZH2/RUNX3 regulatory axis.

\section{Materials and Methods}

Materials. Recombinant human BDNF was obtained from Alomone Laboratories (Jerusalem, Israel). Entinostat (MS-275, pyridine-3ylmethyl$N$-[[4-[(2-aminophenyl) carbamoyl] phenyl] methyl] carbamate) and valpromide were obtained from Santa Cruz Biotechnology (Dallas, TX). MC1568 (3-[5-(3-(3-fluorophenyl)-3oxopropen-1-yl)-1-methyl-1H-pyrrol2-yl]- $N$-hydroxy-2-ropenamide) was from SelleckChem (Houston, TX).
3-Deazaneplanocin A hydrochloride (DZNep), romidepsin (depsipeptide), PCI-34051 (1-(4-methoxybenzyl)- $N$-hydroxy-1H-indole-6-carboxamide), and tubacin (N1-[4-[(2R,4R,6S)-4-[[(4,5-diphenyl-2-oxazolyl) thio]methyl]6-[4-(hydroxymethyl) phenyl]-1,3-dioxan-2-yl]phenyl]-N8-hydroxyoctanediamide) were from MedChem Express Europe (Sollentuna, Sweden). VPA, trichostatin A, sodium butyrate, and 4',6-diamidino2phenylindole dihydrochloride (DAPI) were from Sigma-Aldrich (St. Louis, MO).

Cell Culture. Human neuroblastoma cell lines SH-SY5Y and LAN-1 were obtained from the European Cell Culture Collection (Salisbury, UK) and grown in Ham's F12/MEM medium (1:1) supplemented with $2 \mathrm{mM}$ L-glutamine, $1 \%$ nonessential amino acids, $10 \%$ fetal calf serum (FCS), and $100 \mathrm{U} / \mathrm{ml}$ penicillin- $100 \mu \mathrm{g} / \mathrm{ml}$ streptomycin (Invitrogen, Carlsbad, CA). The Kelly cell line was obtained from Cell Lines Services GmbH (Eppelheim, Germany) and grown in RPMI 1640 medium supplemented with $2 \mathrm{mM} \mathrm{L}$-glutamine and 10\% FCS and $100 \mathrm{U} / \mathrm{ml}$ penicillin- $100 \mu \mathrm{g} / \mathrm{ml}$ streptomycin. Cells were maintained at $37^{\circ} \mathrm{C}$ in a humidified atmosphere of $5 \% \mathrm{CO}_{2}$ in air.

Cells were differentiated by exposure for 6 to 7 days to all-trans retinoic acid (RA) (Sigma-Aldrich) dissolved in dimethyl sulfoxide. The final RA concentration $(10 \mu \mathrm{M})$ was obtained by dilution of stock solution in the cell culture medium. The medium was renewed every 48 hours and during the RA treatment care was taken to avoid cell culture exposure to light.

Primary Cultures of Mouse Cortical Neurons. CD-1 mice were obtained from Envigo RMS S.r.l. (S. Pietro al Natisone, Udine, Italy). Primary cultures of cortical neurons were prepared from 1-day-old mice of mixed sexes as previously described (Dedoni et al., 2010). Cells were plated on six-well plates precoated with $0.01 \%$ poly-L-lysine (Sigma-Aldrich) at the density of $\sim 1 \times 10^{6}$ cells/well and cultured in Neurobasal-A medium (Gibco-Thermo Fisher Scientific, Waltham, MA) supplemented with B-27. Cultures were used 5-7 days after plating and contained $\sim 90 \%$ neurons as assessed by immunofluorescence staining with anti-neurofilament 160/200 (NF160/200) and antiglial fibrillary acidic protein antibodies (Sigma-Aldrich). Experiments were performed according to the recommendations of the European Commission (EU Directive 2010/63/EU for animal experimentation) and were approved by the Institutional Ethical Committee.

Cell Treatment. Unless otherwise indicated, RA-treated neuroblastoma cells were washed with PBS and incubated in medium containing $1 \%$ FCS. Cultured cortical neurons were washed and kept in Neurobasal-A medium without B-27 supplement. Cells were treated with test agents as indicated at $37^{\circ} \mathrm{C}$ in a humidified atmosphere of $5 \% \mathrm{CO}_{2}$ in air. Control samples received an equal amount of vehicle. Thereafter, cells were washed and scraped into ice-cold lysis buffer containing PBS, $0.1 \%$ SDS, $1 \%$ Nonidet P-40, $0.5 \%$ sodium deoxycholate, $2 \mathrm{mM}$ EDTA, $2 \mathrm{mM}$ EGTA, $4 \mathrm{mM}$ sodium pyrophosphate, $2 \mathrm{mM}$ sodium orthovanadate, $10 \mathrm{mM}$ sodium fluoride, $20 \mathrm{nM}$ okadaic acid, $1 \mathrm{mM}$ phenylmethylsulphonyl fluoride (PMSF), $0.5 \%$ phosphatase inhibitor cocktail 3, and $1 \%$ protease inhibitor cocktail (SigmaAldrich) (RIPA buffer). The samples were sonicated for 5 seconds in ice-bath, and aliquots of cell extracts were taken for protein determination by the Bio-Rad protein assay (Bio-Rad Laboratory, Hercules, CA).

Isolation of Cell Nuclei. SH-SY5Y cells grown in $100 \mathrm{~mm}$ Petri dishes were treated with the agents as indicated, washed with ice-cold PBS (pH 7.4), and scraped in an ice-cold lysis buffer containing PBS, $10 \mathrm{mM}$ Tris-HCl, $2 \mathrm{mM} \mathrm{MgCl}_{2}, 10 \mathrm{mM} \mathrm{NaCl}, 0.5 \mathrm{mM}$ EGTA, $2 \mathrm{mM}$ sodium orthovanadate, $10 \mathrm{mM}$ sodium fluoride, $1 \mathrm{mM}$ PMSF, $0.05 \%$ Nonidet, $0.5 \%$ phosphatase inhibitor cocktail 3 , and $1 \%$ protease inhibitor cocktail ( $\mathrm{pH} 7.4$ ). Cell lysates were centrifuged at $3000 \mathrm{~g}$ for 10 minutes at $4^{\circ} \mathrm{C}$, the supernatant was collected and centrifuged at $24,000 \mathrm{~g}$ for 20 minutes to obtain a cytosolic fraction. The pellets were washed three times in ice-cold washing buffer containing $10 \mathrm{mM} \mathrm{1,4-}$ piperazinediethanesulfonic acid/KOH, $300 \mathrm{mM}$ sucrose, $2 \mathrm{mM} \mathrm{MgCl}$, $10 \mathrm{mM} \mathrm{NaCl}, 0.5 \mathrm{mM}$ EGTA, $2 \mathrm{mM}$ sodium orthovanadate, $10 \mathrm{mM}$ sodium fluoride, $1 \mathrm{mM}$ PMSF, $0.5 \%$ phosphatase inhibitor cocktail 3, and $1 \%$ protease inhibitor cocktail ( $\mathrm{pH} 7.4$ ) and layered over a cushion of $1 \mathrm{ml}$ of sucrose buffer containing $1 \mathrm{M}$ sucrose, $2 \mathrm{mM}$ sodium 
orthovanadate, $10 \mathrm{mM}$ sodium fluoride, $1 \mathrm{mM}$ PMSF, $0.5 \%$ phosphatase inhibitor cocktail 3 and $1 \%$ protease inhibitor cocktail. Following centrifugation at $3000 \mathrm{~g}$ for 10 minutes at $4^{\circ} \mathrm{C}$, the nuclei present in the pellets were washed and the proteins were extracted by incubating the nuclei on ice for 30 minutes in a buffer containing $20 \mathrm{mM} 4-(2-$ hydroxyethyl)-1-piperazineethanesulfonic acid/ $\mathrm{NaOH}, 300 \mathrm{mM} \mathrm{NaCl}$, $2 \mathrm{mM} \mathrm{MgCl}_{2}, 0.2 \mathrm{mM}$ EDTA, $2 \mathrm{mM}$ sodium orthovanadate, $10 \mathrm{mM}$ sodium fluoride, $1 \mathrm{mM}$ PMSF, $0.5 \%$ phosphatase inhibitor cocktail 3 and $1 \%$ protease inhibitor cocktail $(\mathrm{pH} 7.9)$. Following centrifugation at $24,000 \mathrm{~g}$ for 10 minutes at $4^{\circ} \mathrm{C}$, the nuclear extracts were mixed with sample buffer and analyzed by Western blot.

Transfection of Small Interfering RNA. SH-SY5Y cells were transfected with either $50 \mathrm{pmol} / \mathrm{ml}$ of control small interfering RNA (siRNA)-A (sc-37007; Santa Cruz Biotechnology), HDAC1 siRNA (sc-29343; Santa Cruz Biotechnology), or RUNX3 siRNA (sc-37679; Santa Cruz Biotechnology) duplexes using Lipofectamine RNAiMAX (Invitrogen) as a transfection reagent. Control siRNA consisted of a nontargeting sequence. Cells grown in six-well plates were incubated in antibiotic-free medium for 24 hours. The medium was renewed and the cells were incubated with siRNA duplexes for 4 to 5 hours at $37^{\circ} \mathrm{C}$. Thereafter, the medium was replaced by the growth medium, and the cells were analyzed 48 hours posttransfection. To determine transfection efficiency, parallel samples were transfected with fluoresceinconjugated control siRNA-A (sc-36869; Santa Cruz Biotechnology). An efficiency of $50 \%-60 \%$ was obtained in six separate experiments.

RNA Extraction and Quantitative Polymerase Chain Reaction. SH-SY5Y cells were incubated in a medium containing $1 \%$ FCS and treated for 24 hours with either vehicle or $1 \mathrm{mM}$ VPA. Thereafter, the cells were washed with PBS and total RNA was isolated by using TRIzol reagent (Invitrogen) with the PureLink RNA mini kit as specified by the manufacturer's protocol. After RNA purification a Turbo DNase (Ambion by Life Technologies) digestion was performed. The purity and quantity of the RNA isolated were determined by measuring UV absorbance at 260 and $280 \mathrm{~nm}$.

First-strand cDNA synthesis was performed using $2 \mu \mathrm{g}$ of total RNA, following the manufacturer's protocol (SuperScript VILO cDNA synthesis kit; Invitrogen). Two-hundred nanograms of cDNA for reaction was used for quantitative polymerase chain reaction amplification with SYBR Green PCR Master Mix (Applied Biosystems). The quantitative polymerase chain reaction analysis was carried out on a real-time PCR system (StepOne; Applied Biosystems) under the following conditions: an initial holding stage at $95^{\circ} \mathrm{C}$ for 10 minutes was followed by 45 cycles: denaturation at $95^{\circ} \mathrm{C}$ for 15 seconds, primer annealing and extension at $60^{\circ} \mathrm{C}$ for 1 minute, and a dissociation curve to the end of a real time run (melt curve $95^{\circ} \mathrm{C}$ for 15 seconds, $60^{\circ} \mathrm{C}$ for 1 minute and $95^{\circ} \mathrm{C}$ for 15 seconds). The PCR primers used were: human TrkB (NCBI accession no. NM_006180.3) forward ACAGTCAGCTCAAGCCAGACAC, reverse GTCCTGCTCAGGACAGAGGTTA; human $\beta$-actin (NCBI accession number NM_001101.3) forward AGCCTCGCCTTTGCCGATCCG, reverse CATGCCGGAGCCGTTGTCGAC. The comparative $\mathrm{Ct}$ values method was used to calculate the relative quantity of TrkB expression. For both control and VPA-treated samples, the $\beta$-actin $\mathrm{Ct}$ values were subtracted from the TrkB Ct values to obtain the $\Delta \mathrm{Ct}$ value. $\Delta \Delta \mathrm{Ct}$ values were obtained according to $\Delta \mathrm{Ct}$ (VPA-treated samples) $-\Delta \mathrm{Ct}$ (control samples). The relative quantity was calculated according to $2^{-\Delta \Delta \mathrm{Ct}}$.

Biotinylation of Surface Proteins. Surface biotinylation of cell proteins was performed as previously described (Dedoni et al., 2012). Differentiated SH-SY5Y cells were grown in $100 \mathrm{~mm}$ plates and treated at confluency with either vehicle or VPA $(1 \mathrm{mM})$ for 24 hours in medium containing $1 \%$ FCS. Thereafter the cells were washed three times with ice-cold PBS ( $\mathrm{pH} 8.0$ ) and incubated for 45 minutes at $4^{\circ} \mathrm{C}$ with the cell impermeable biotinylating agent sulfosuccinimidyl-6(biotin-amido)hexanoate $(0.50 \mathrm{mg} / \mathrm{ml})$ (Pierce, Rockford, IL). Thereafter, the medium was aspirated and the cells washed three times with ice-cold PBS containing $20 \mathrm{mM}$ glycine. Cells were then solubilized by incubation for 60 minutes at $4^{\circ} \mathrm{C}$ in RIPA buffer supplemented with $1 \%$ Triton $\times 100$. Cell extracts were centrifuged at $14,000 \mathrm{~g}$ for
5 minutes at $4^{\circ} \mathrm{C}$, and the supernatants were incubated overnight with streptavidin-conjugated agarose beads with continuous rotation at $4^{\circ} \mathrm{C}$. The samples were then centrifuged to obtain a supernatant and a pellet fraction containing the plasma membrane-associated proteins. The agarose beads were washed three times with ice-cold Tris buffer containing $10 \mathrm{mM}$ Tris-HCl (pH 7.5), $2.5 \mathrm{mM}$ EDTA, $150 \mathrm{mM} \mathrm{NaCl}$, and $1 \%$ Triton $\times 100$, followed by two washes with $10 \mathrm{mM}$ Tris-HCl (pH 7.5), $2.5 \mathrm{mM}$ EDTA, $500 \mathrm{mM} \mathrm{NaCl}$, and $0.1 \%$ Triton $\times 100$ and one final wash with $50 \mathrm{mM}$ Tris- $\mathrm{HCl}(\mathrm{pH} 7.5)$. The pellets were then mixed with sample buffer and incubated 2 minutes at $100^{\circ} \mathrm{C}$. The proteins were then analyzed by Western blot.

Western Blot Analysis. Cell proteins were separated by SDSpolyacrylamide gel and electrophoretically transferred to polyvinylidene difluoride membranes (Amersham Biosciences, Piscataway, NJ). Membranes were blocked, washed, and incubated overnight at $4^{\circ} \mathrm{C}$ with one of the following primary antibodies: TrkB (cat. no. 4603; Cell Signaling Technology, Danvers, MA) (1:1000), TrkA (cat. no. 2508; Cell Signaling Technology) (1:1000), TrkC (cat. no. 3376; Cell Signaling Technology) (1:1000), phospho-TrkA (Tyr674/675)/TrkB (Tyr706/ 707) (cat. no. 4621; Cell Signaling Technology) (1:1000), p75NTR (cat. no. 8238; Cell Signaling Technology) (1:1000), synapsin-1 (cat. no. 5297; Cell Signaling Technology) (1:2000), pan-cadherin (cat. no. 4073; Cell Signaling Technology) (1:1000), phospho-S6 ribosomal protein (Ser235/ 236) (cat. no. 4856; Cell Signaling Technology), S6 ribosomal protein (cat. no. sc-74459; Santa Cruz Biotechnology) (1:1000), phospho-Akt (Thr308) (cat. no. 2965; Cell Signaling Technology) (1:1000), Akt1/2/3 (cat. no. sc-8312; Santa Cruz Biotechnology) (1:1000), phospho-glycogen synthase kinase (GSK)-3 $\beta$ (Ser9) (cat, no. 9356; Cell Signaling Technology) (1:1000), GSK-3 $\beta$ (cat no. sc-9166; Santa Cruz Biotechnology) (1:2000), extracellular signal-regulated kinases 1 and 2 (ERK1/2) (cat no. 9102; Cell Signaling Technology) (1:1000), phospho-ERK1 (Thr202/Tyr204)/ERK2 (Thr185/Tyr187) (cat. no. RA15002; Neuromics, Northfield, MN) (1:15,000), phospholipase C $\gamma 1$ (PLC $\gamma 1$ ) (cat no. 2822; Cell Signaling Technology) (1:1000), phospho-PLC $\gamma 1$ (Tyr783) (cat. no. 2821; Cell Signaling Technology) (1:1000), cleaved-poly(ADPribose) polymerase (PARP) (Asp214) (cat. no. 5625; Cell Signaling Technology) (1:1000), PARP (cat. no. 9542; Cell Signaling Technology) (1:1000), enhancer of zeste homolog 2 (EZH2) (cat. no. 5246; Cell Signaling Technology) (1:2000), RUNX3/AML2 (cat. no. 9647; Cell Signaling Technology) (1:1000), postsynaptic density 95 (PSD95) (cat. no. 3450; Cell Signaling Technology) (1:2000), GluR1 (cat no.2040; Tocris Cookson Ltd., Avonmouth, UK) (1:1000), HDAC1 (cat. no. sc-81598; Santa Cruz Biotechnology) (1:5000), histone H3 (cat. no. GTX122148; GeneTex Inc., Irvine, CA) (1:2000), histone H3 (acetyl-Lys9/Lys14) (cat no. GTX122648; GeneTex Inc.), (1:1000), MAP2 (cat. no. 188011; Synaptic Systems GmbH, Gottingen, Germany) (1:1000), actin (cat no. A2066; Sigma-Aldrich) (1:3000), actin (cat. no. A5441; Sigma-Aldrich) (1:20,000), andglyceraldehyde 3-phosphate dehydrogenase (GAPDH) (cat. no. 247002; Synaptic Systems GmbH) (1:1000). Thereafter, the membranes were washed and incubated with an appropriate horseradish peroxidaseconjugated secondary antibody (Santa Cruz Biotechnology). Immunoreactive bands were detected by using Clarity Western ECL substrate (Bio-Rad Laboratory.) and ECL Hyperfilm (Amersham). The size of immunoreactive bands was determined by using molecular weight standards detected with an ECL suitable antibody (cat. no. sc-2035; Santa Cruz Biotechnology) (1:1000). Band densities were determined using Image Scanner III (GE Healthcare, Milan, Italy) and NIH ImageJ software (US National Institutes of Health, Bethesda, MA). The optical density of the phosphorylated protein bands was normalized to the density of the corresponding total protein. For analysis of PARP, the formation of the cleaved protein was normalized to the level of the uncleaved PARP determined in the same samples. For the remaining proteins, the densitometric values were normalized to the levels of either actin, GAPDH, or subcellular fraction marker, as indicated.

Immunofluorescence Analysis. Differentiated SH-SY5Y cells grown on poly-L-lysine-coated coverslips were treated with the test agents as indicated in the text, washed, and fixed in $4 \%$ paraformaldehyde for 
30 minutes at room temperature. For analysis of TrkB, nonpermeabilized cells were blocked with $3 \%$ BSA and $1 \%$ normal goat serum and incubated overnight with an antibody directed against an extracellular domain of TrkB (1:50) (cat. no. ANT-019; Alomone Laboratories). For analysis of neuritogenesis and detection of cleavedcaspase 3 , cells were permeabilized with $0.2 \%$ Triton X-100, blocked, and incubated overnight at $4^{\circ} \mathrm{C}$ with mouse anti-actin (cat. no. A5441; Sigma-Aldrich) (1:5000) and rabbit anti-cleaved caspase-3 (cat. no. 9661; Cell Signaling Technology) (1:200), respectively.

Cells were then incubated with an appropriate Alexa-Fluor488- or Alexa-Fluor594-conjugated secondary antibody (Invitrogen-Molecular Probes), and cell nuclei were stained with $0.1 \mu \mathrm{g} / \mathrm{ml}$ 4',6-diamidino2phenylindole. Cells were analyzed with an Olympus BX61 microscope equipped with a F-View II CCD-camera by using either a $\times 40$ or a $\times 60$ objective lens. Digital images were acquired using constant camera settings within each experiment and were analyzed using the program Cell P (Olympus Soft Imaging Solutions, Homburg, Germany). At least 15 fields were randomly selected for each sample, and only cells showing an unobstructed nucleus or soma were considered.

For quantification of neuritogenesis, at least 10 fields were analyzed for each sample run in triplicate and in each field the cell bodies and neurites were counted. Processes with length equal to two or more cell body diameters were scored as neurites. The ratio of neurites to cell bodies was calculated and expressed as mean \pm S.E.M. Four separate preparations of SH-SY5Y cells were examined. For analysis of TrkB and cleaved caspase 3 expression, the average pixel intensity was measured within the region of the cell soma or the nucleus and in an adjacent area, which was used as background value. Cells were deemed to be positive if the average pixel intensity was equal or above a threshold value corresponding to 1 S.D. above the average pixel intensity of the respective control samples (Dedoni et al., 2017). No labeling was detected in samples treated without primary antibodies. For each experiment, four separate preparations of SH-SY5Y cells were analyzed by an investigator unaware of the treatment.

Statistical Analysis. Results are reported as the mean \pm S.E.M. of $N$ independent experiments. Statistical analysis was performed by using the program Graph Pad Prism 5 (San Diego, CA). Unless otherwise indicated, data are expressed as percentage or fold stimulation of control, which was included in each independent experiment. The control group was set as 100 or 1 , with a variance obtained by expressing each control value as a percentage of the mean of the raw values of the control group. In the experiments in which control values were equal to zero, values of experimental groups were expressed as a percentage of the maximal effect set as 100 . The variance of this value was determined in the same manner as for the control group. Statistical analysis was performed by either Student's unpaired $t$ test or one-way analysis of variance followed by Newman-Keuls post hoc test as appropriate. A value of $P<0.05$ was considered to be statistically significant.

\section{Results}

VPA Downregulates TrkB Expression in RADifferentiated Neuroblastoma Cells. In agreement with previous studies (Kaplan et al., 1993; Encinas et al., 1999; Dedoni et al., 2012), Western blot analysis of TrkB expression in RAdifferentiated SH-SY5Y cells detected the presence of an immunoreactive band of $145 \mathrm{kDa}$ representing the catalytic tyrosine kinase full-length isoform of TrkB (TrkB-FL) and an additional broader band of $\sim 95 \mathrm{kDa}$, which corresponded to the molecular mass of the truncated TrkB (TrkB-T) isoforms TrkB.T1 and TrkB.Shc lacking the tyrosine kinase domain (Fig. 1A) (Klein et al., 1990; Stoilov et al., 2002). Prolonged (24 hours) cell treatment with VPA ( $1 \mathrm{mM})$ reduced the expression levels of the $145-$ and $95-\mathrm{kDa}$ isoforms by $63 \% \pm 6 \%$ and $47 \% \pm$ $5 \%(P<0.001, N=6)$, respectively (Fig. 1A). Quantitative RT-PCR analysis indicated that VPA reduced the level of TrkB mRNA by $45 \% \pm 5 \%(P<0.001, N=3)$ (Fig. 1B).

We also investigated the effects of VPA on other neurotrophin receptors whose expression is known to be enhanced by RA treatment of neuroblastoma cells. Prolonged exposure
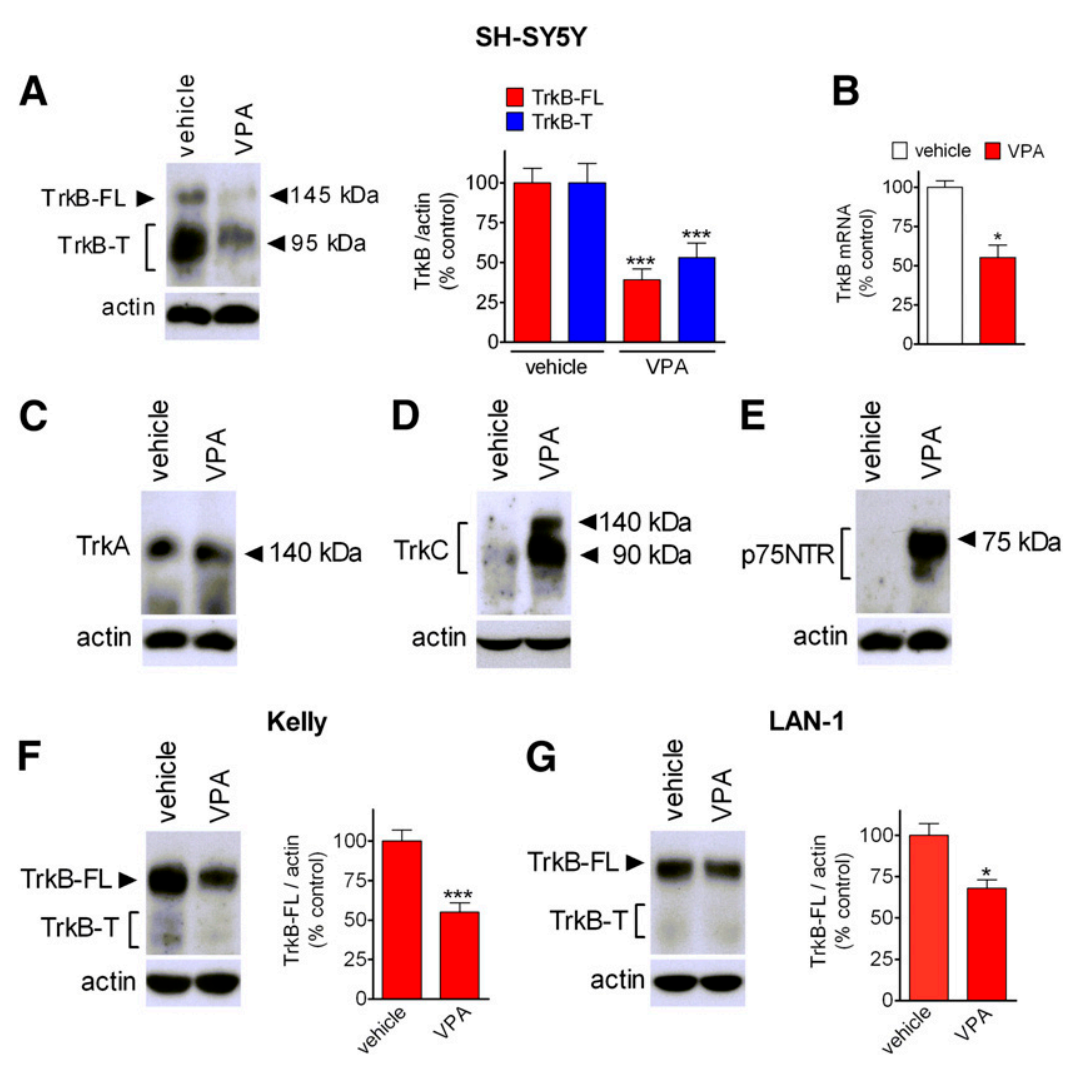

Fig. 1. VPA downregulates TrkB expression in RAdifferentiated neuroblastoma cells. (A) SH-SY5Y cells were incubated for 24 hours with either vehicle or $1 \mathrm{mM}$ VPA, and the expression of full length (TrkB-FL) and truncated (TrkB-T) isoforms of TrkB was analyzed by Western blot and normalized to actin levels. Values are the mean \pm S.E.M. of six independent experiments. (B) Quantitative real-time RT-PCR analysis of TrkB mRNA in SH-SY5Y cells treated for 24 hours with either vehicle or $1 \mathrm{mM}$ VPA. Values are the mean \pm S.E.M. of three independent determinations. (C-E) SH-SY5Y cells were treated as in (A), and the expression of TrkA (C), TrkC (D), and p75NTR (E) was determined in cell lysates. (F and G) Kelly (F) and LAN-1 (G) cells were treated for 24 hours with either vehicle or VPA $(1 \mathrm{mM})$. Cell lysates were then analyzed for TrkB expression. Values are the mean \pm S.E.M. of four (Kelly) and seven (LAN-1) experiments. $* P<0.05$; $* * * P<$ 0.001 vs. control (vehicle) by Student's $t$ test. 

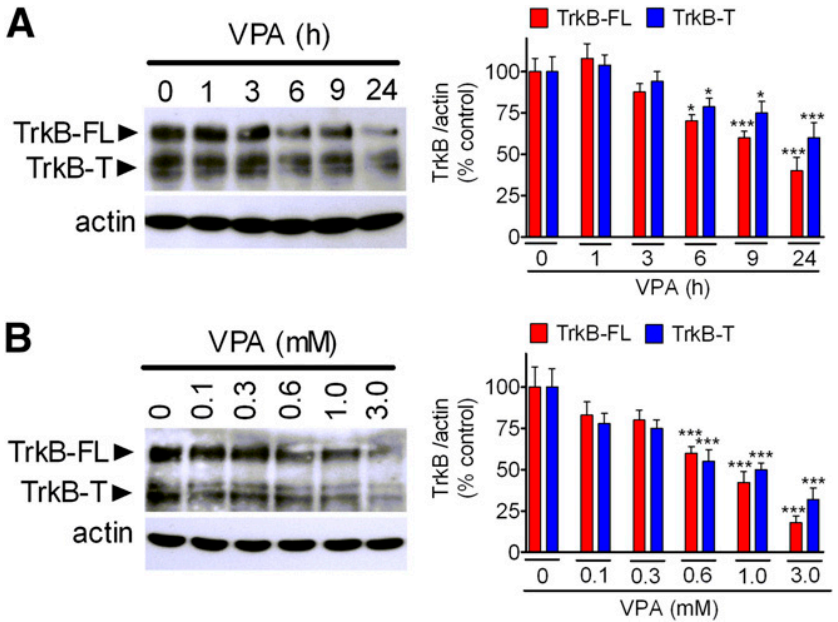

Fig. 2. Time- and concentration-dependent inhibition of TrkB expression by VPA. (A) SH-SY5Y cells were incubated in the presence of $1 \mathrm{mM}$ VPA for the indicated periods of time. Zero time samples were treated with vehicle and used as control. (B) SH-SY5Y cells were incubated for 24 hours in the presence of either vehicle or VPA at the indicated concentrations. Cell lysates were analyzed for TrkB expression. Values are the mean \pm S.E.M. of four experiments. ${ }^{*} P<0.05$; $* * * P<0.001$ vs. control (vehicle) by analysis of variance (ANOVA) followed by Newman-Keuls post hoc test.

of differentiated SH-SY5Y cells to VPA (1 mM) failed to significantly affect the protein levels of TrkA, the receptor of nerve growth factor (Fig. 1C), but markedly enhanced the expression of the full-length and truncated isoforms of TrkC, the receptor for neurotrophin 3 (Fig. 1D), and the common neurotrophin receptor p75NTR (Fig. 1E). Densitometric values of vehicleand VPA-treated samples (expressed as fold changes \pm S.E.M. with respect to vehicle set to 1.0$)$ were: TrkA $1.28 \pm 0.15(P>0.05$, $N=4)$; TrkC-FL $20 \pm 3$, TrkC-T $6.5 \pm 1.0(P<0.001 N=3)$; p75NTR $50 \pm 8(P<0.001, N=6)$.

We examined the effects of VPA on TrkB expression in two additional human neuroblastoma cell lines, LAN-1 and Kelly, which, differently from SH-SY5Y cells, display MYCN oncogene amplification. Both Kelly and LAN-1 cells predominantly expressed the TrkB-FL isoform upon RA exposure (Supplemental Fig. 1). Treatment with VPA (1 mM) for 24 hours decreased the levels of TrkB-FL by $46 \% \pm 5 \%(P<0.001, N=4)$ and $32 \% \pm 4 \%(P<0.05, N=7)$ in Kelly and LAN-1 cells, respectively (Fig. 1, F and G).

The VPA inhibition of TrkB expression was time dependent, being significant after 6 hours of treatment and increasing progressively up to 24 hours (Fig. 2A). The inhibitory effect was also concentration dependent, being absent at 0.1 and $0.3 \mathrm{mM}$ VPA and increasing as the drug concentrations were elevated from 0.6 to $3 \mathrm{mM}$ (Fig. 2B).

To investigate whether VPA affected the plasma membrane expression of TrkB, surface protein biotinylation experiments were performed in SH-SY5Y cells. As shown in Fig. 3A, exposure to VPA $(1 \mathrm{mM})$ reduced the cell surface levels of TrkB-FL and TrkB-T by $65 \% \pm 6 \%$ and $47 \% \pm 5 \%(P<0.001$, $N=4$ ), respectively, compared with vehicle-treated cells. Moreover, immunofluorescence analysis of TrkB in nonpermeabilized cells, using an antibody directed against an extracellular domain of the receptor to detect its plasma membrane expression, indicated that VPA treatment decreased the percentage of TrkB-positive cells by $64 \% \pm 4 \%(P<0.001, N=4)$ (Fig. 3B).

VPA Exposure Inhibits BDNF-Induced TrkB Activation and Signaling. BDNF-induced TrkB activation involves the receptor autophosphorylation at distinct tyrosine residues located in the intracytoplasmatic domain, which regulates the receptor activity and allows the docking of adaptor signaling molecules. Exposure of SH-SY5Y cells to VPA (1 mM) induced a marked inhibition of BDNF (1 nM)-induced TrkB phosphorylation at Tyr706/707, which occurs within the activation loop of the catalytic domain and enhances the integral receptor tyrosine kinase activity (Huang and Reichardt, 2003) (Fig. 4A). The inhibitory effect of VPA remained significant when phosphoTrkB levels were normalized to those of total TrkB-FL, indicating that VPA-induced TrkB-FL downregulation was associated with impairment of BDNF-induced receptor activation.

VPA treatment markedly reduced the ability of BDNF to trigger the activation of $\mathrm{PLC} \gamma$ and PI3K/Akt signaling pathways, which are predominantly involved in the promotion of synaptic

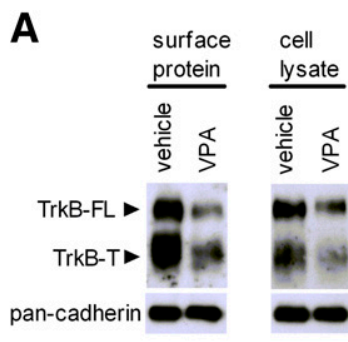

B

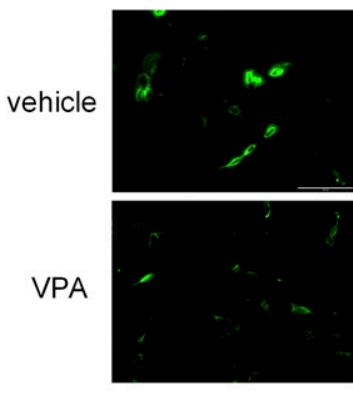

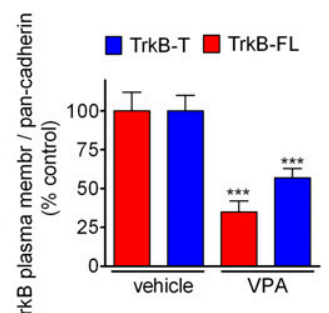

DAPI

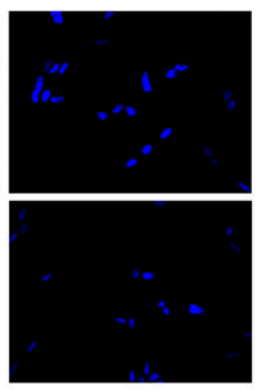

Merged

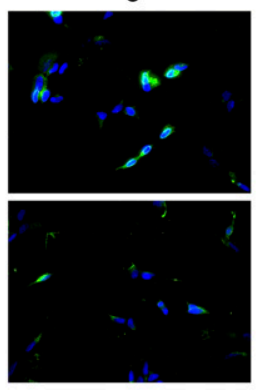

Fig. 3. VPA reduces plasma membrane expression of TrkB. SH-SY5Y cells were treated with either vehicle or VPA ( $1 \mathrm{mM})$ for 24 hours. Cells were then exposed to the cell impermeant biotinylating agent sulfosuccinimidyl6-(biotin-amido)hexanoate and solubilized proteins were precipitated by using streptavidin-conjugated agarose beads. The precipitate (surface protein) and total extract (cell lysate) were analyzed for TrkB by Western blot (A). TrkB levels in the surface protein preparation were normalized to pan-cadherin levels, a plasma membrane (membr) marker. Densitometric values are expressed as percent of control (vehicle) and are the mean \pm S.E.M. of four experiments. (B) TrkB expression was analyzed by immunofluorescence (green color) in nonpermeabilized cells with an antibody directed against an extracellular domain of the receptor. Nuclei were stained in blue with 4',6-diamidino-2phenylindole (DAPI). Values are the mean \pm S.E.M. of four experiments. Scale bar, $25 \mu \mathrm{m}$. ${ }^{* * *} P<0.001$ vs. control (vehicle) by Student's $t$ test. 


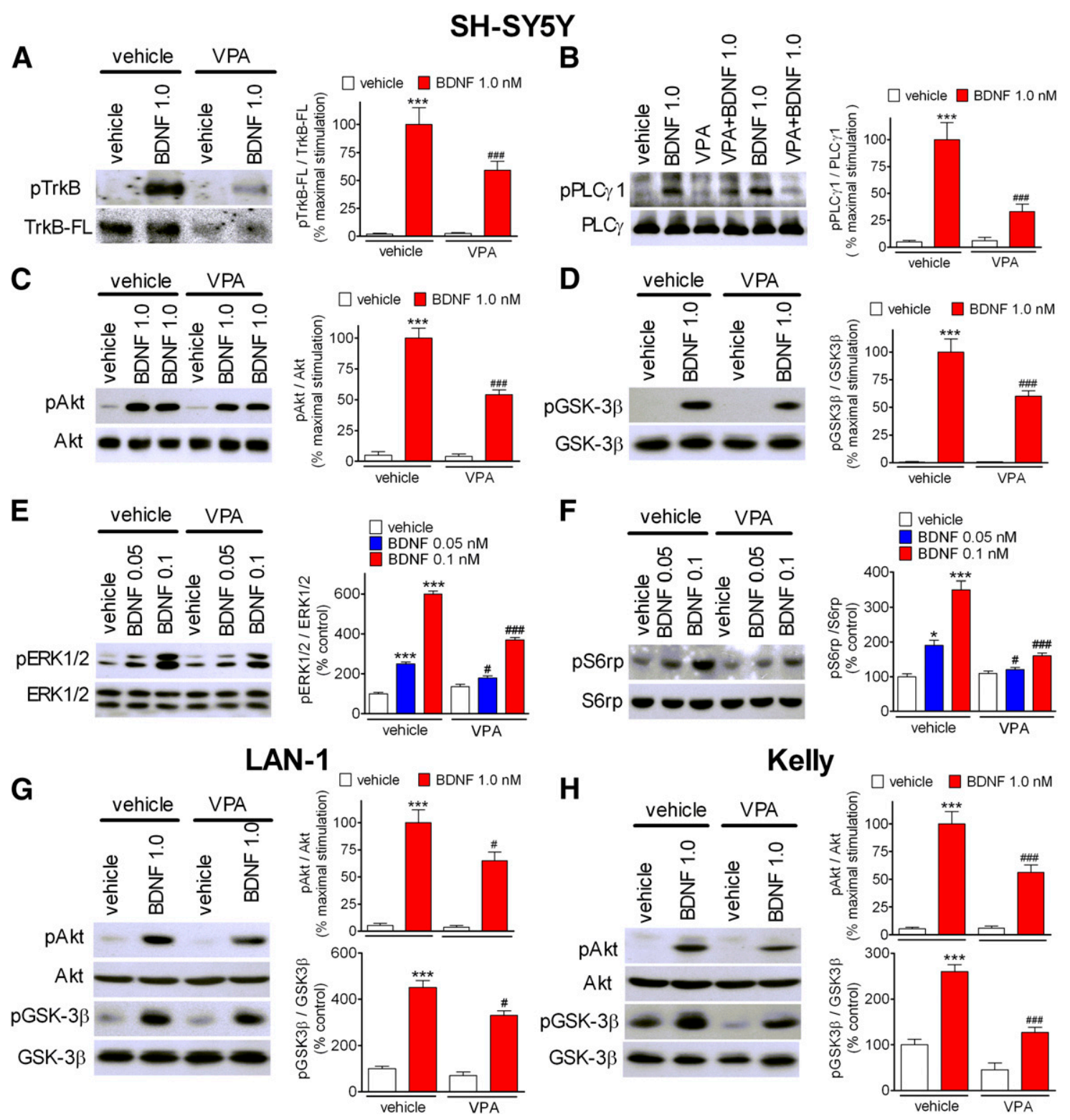

Fig. 4. Inhibition of BDNF-induced TrkB activation and signaling by VPA. (A-D) SH-SY5Y cells were incubated for 24 hours with either vehicle or $1 \mathrm{mM}$ VPA and then exposed for 5 minutes to either vehicle or $1 \mathrm{nM}$ BDNF. Cell lysates were analyzed for the expression of phospho-Tyr706/707-TrkB (pTrkB), total TrkB-FL (A), phospho-Tyr783-PLC $\gamma 1$ (pPLC $\gamma 1$ ), total PLC $\gamma 1$ (B), phospho-Thr308-Akt (pAkt), total Akt (C), phospho-Ser9-GSK-3 $\beta$ (pGSK-3 $\beta$ ), and total GSK-3 $\beta$ (D). Values are the mean \pm S.E.M. of four experiments. (E and F) SH-SY5Y cells were incubated for 24 hours with either vehicle or $1 \mathrm{mM}$ VPA and then exposed for 5 minutes to either vehicle, $0.05 \mathrm{nM}$ BDNF (BDNF 0.05), or 0.1 nM BDNF (BDNF 0.1). Cell lysates were analyzed for phosphoERK1/2 (pERK1/2), total ERK1/2, phospho-Ser235/236-S6 ribosomal protein (pS6rp), and total S6 ribosomal protein (S6rp). Values are the mean \pm S.E.M. of three experiments. (G and H) LAN-1 (G) and Kelly (H) cells were incubated for 24 hours with either vehicle or $1 \mathrm{mM}$ VPA and then exposed for 5 minutes to either vehicle or $1 \mathrm{nM}$ BDNF. Values are the mean \pm S.E.M. of three experiments. $* P<0.05 ; * * * P<0.001 \mathrm{vs}$. control (vehicle); ${ }^{*} P<0.05$; ${ }^{\# \# \# P}<0.001$ vs. BDNF + vehicle by ANOVA followed by Newman-Keuls post hoc test.

plasticity and neuronal survival, respectively (Huang and Reichardt, 2003). In SH-SY5Y, cells pre-exposed to VPA (1 mM) the phosphorylation of PLC $\gamma 1$ at Tyr783, which is essential for neurotrophin-induced PLC $\gamma 1$ activation (Kim et al., 1991), was reduced by $67 \% \pm 7 \%(P<0.001, N=4)$ (Fig. 4B). VPA inhibited the BDNF-induced phosphorylation of Akt at Thr308 by $46 \% \pm 4 \%(P<0.001, N=4)$ (Fig. $4 \mathrm{C})$ and phosphorylation of the Akt substrate GSK-3 $\beta$ at Ser9 by $40 \% \pm 5 \%(P<0.001$, $N=4$ ) (Fig. 4D).

Activation of ERK1/2 constitutes another major signaling pathway activated by the BDNF-TrkB receptor complex. As shown in Fig. 4, E and F, in SH-SY5Y cells, BDNF (0.05 and
$0.1 \mathrm{nM}$ ) stimulated the phosphorylation of ERK1/2 and the downstream ERK1/2 target S6 ribosomal protein in a concentration dependent manner, and these responses were markedly reduced in cells pre-exposed to VPA.

Significant inhibitory effects of VPA $(1 \mathrm{mM})$ on BDNF-induced phosphorylation of Akt and GSK-3 $\beta$ were also observed in LAN-1 and Kelly cells (Fig. 4, G and $\mathrm{H}$ ).

VPA Reduces the Proneurotrophic and Antiapoptotic Activities of BDNF. The induction of TrkB following treatment with RA allows neuroblastoma cells to respond to BDNF with neuronal differentiation, as indicated by the appearance of morphologic changes and the enhanced expression of neuronal 
A
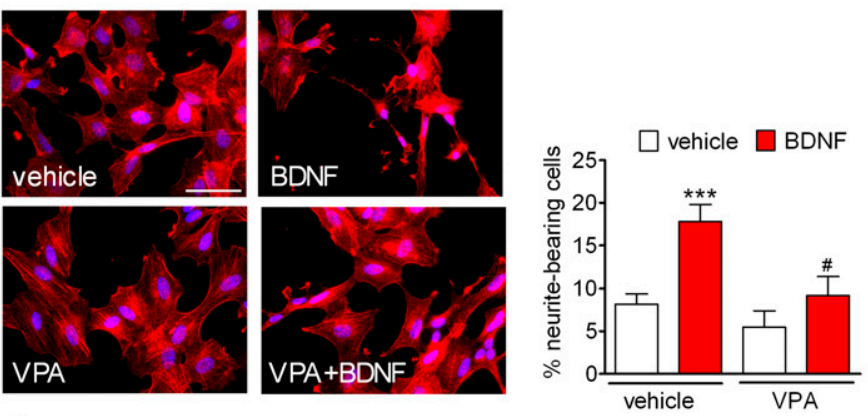

B
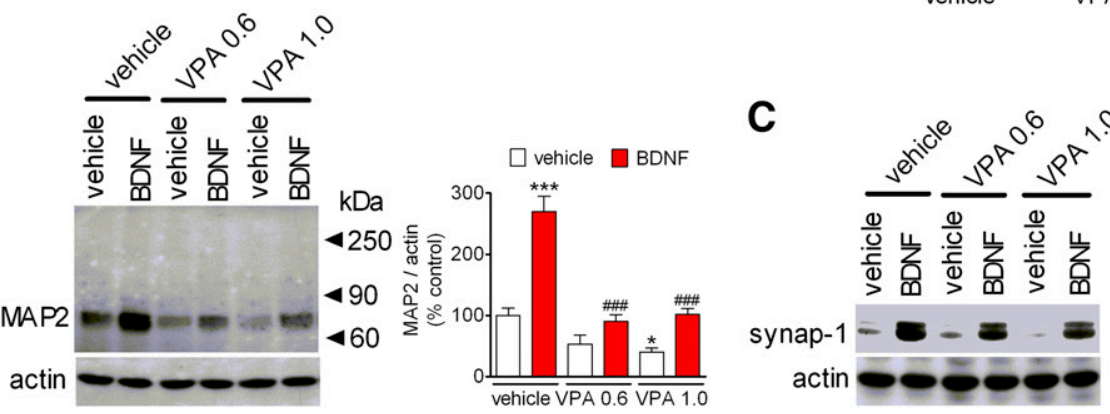

synap-1

actin
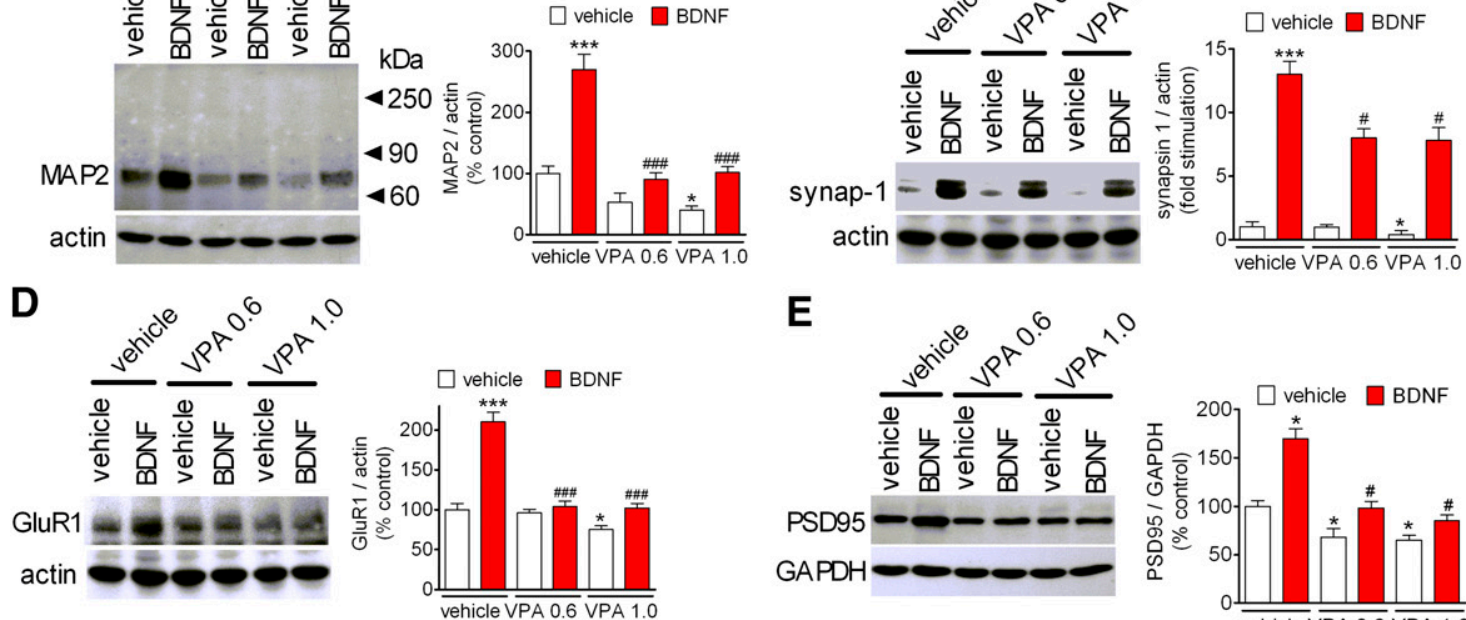

E

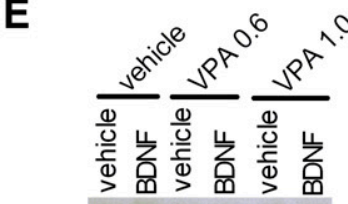

PSD95

GAPDH
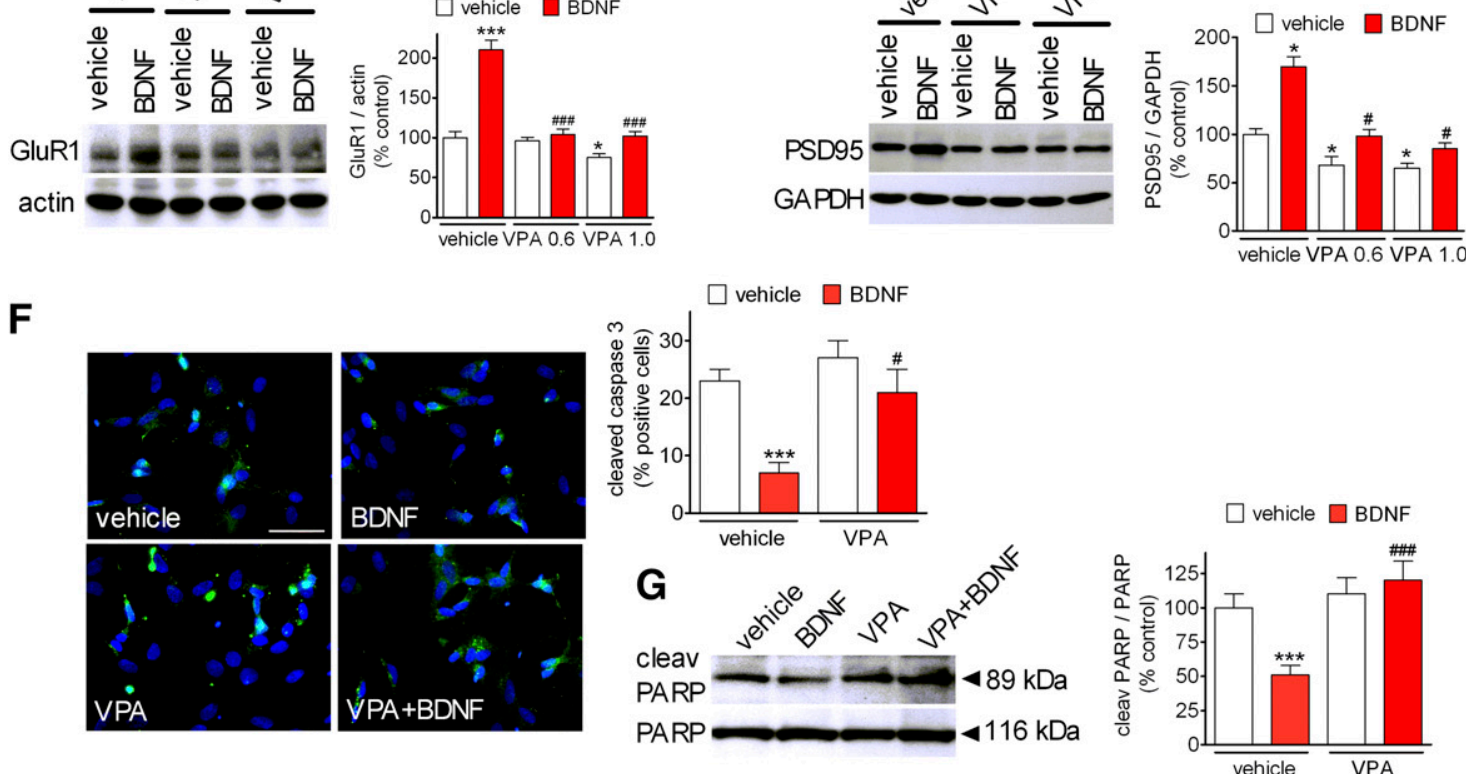

Fig. 5. VPA curtails BDNF-induced proneurotrophic and antiapoptotic responses. (A) SH-SY5Y cells were preincubated for 24 hours with either vehicle or $1 \mathrm{mM}$ VPA and then exposed for 48 hours to either vehicle or $1.0 \mathrm{nM}$ BDNF. Cells were fixed and stained with an antiactin antibody (red color). Nuclei were stained in blue with DAPI. For each sample, the number of neurite-bearing cells was determined and calculated as percent of total cells. Values are the mean \pm S.E.M. of three separate experiments. (B-E) Cells were preincubated for 24 hours with either vehicle, VPA 0.6 mM (VPA 0.6), or VPA $1.0 \mathrm{mM}$ (VPA 1.0) and then exposed to either vehicle or $1 \mathrm{nM}$ BDNF for 48 hours. Cell lysates were analyzed for the expression of MAP2 (B), synapsyn-1 (synap-1) (C), GluR1 (D), and postsynaptic density 95 (PSD95) (E). Values are the mean \pm S.E.M. of four experiments. (F) SH-SY5Y cells were pretreated for 24 hours with either vehicle or $1 \mathrm{mM}$ VPA and then incubated in serum-free medium in the absence and in the presence of $1 \mathrm{nM}$ BDNF for 48 hours. Cells were fixed and analyzed by immunofluorescence for cleaved caspase 3 expression (green color). Cell nuclei were stained with DAPI. For each sample, the number of cleaved caspase 3-positive cells was determined and calculated as percent of total cells. Values are the mean \pm S.E.M. of three experiments. (G) SH-SY5Y cells were treated as indicated in (F) and cell lysates were analyzed for cleaved PARP (cleav PARP) and uncleaved PARP

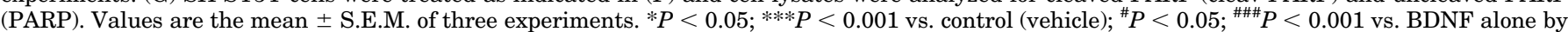
ANOVA followed by Newman-Keuls post hoc test.

markers (Kaplan et al., 1993; Encinas et al., 1999; Dedoni et al., 2012). SH-SY5Y cells treated with RA mostly showed large cell bodies with a polygonal shape and short neurites (Fig. 5A), whereas the exposure of the cells to BDNF $(1 \mathrm{nM})$ for 48 hours increased the percentage of cells with a smaller and rounded cell body and long neuritic processes. Cells treated with
VPA $(1 \mathrm{mM})$ for 72 hours displayed a morphology similar to that of vehicle-treated cells. However, when the cells were preincubated for 24 hours with VPA and then exposed to BDNF and VPA for 48 hours, there was a significant reduction in the percentage of neurite-bearing cells compared with cells treated with the neurotrophin alone (Fig. 5A). 
The microtubule-associated protein 2 (MAP2) is a major component of the neuronal cytoskeleton and comprises proteins with high molecular mass (MAP2A and MAP2B, $\sim 270 \mathrm{kDa}$ ) and low molecular mass (MAP2C, $\sim 70 \mathrm{kDa}$ ) (Garner and Matus, 1988). Western blot analysis of MAP2 expression in RA-differentiated SH-SY5Y cells with an antibody that recognizes all three MAP2 forms detected a single immunoreactive band of $\sim 70 \mathrm{KDa}$, indicating the presence of MAP2C. Prolonged exposure to BDNF increased the expression of MAP2 by about threefold, and this effect was prevented by cell pretreatment with 0.6 and $1 \mathrm{mM}$ VPA (Fig. 5B). Cell treatment with BDNF also enhanced the steady-state levels of synapsin-1 proteins (Fig. 5C), which regulate synaptogenesis and neurotransmitter release (Cesca et al., 2010), the glutamate AMPA receptor subunit GluR1 (Fig. 5D), and the scaffolding protein postsynaptic density 95 (Fig. 5E). Cell pretreatment with VPA (0.6 and $1 \mathrm{mM})$ significantly curtailed these neurotrophic responses to BDNF.

Prolonged serum withdrawal induces apoptotic cell death of SH-SY5Y cells (Macleod et al., 2001; Dedoni et al., 2017). Addition of BDNF ( $1 \mathrm{nM})$ to the serum-free medium protected against apoptosis, as indicated by the significant decrease in the percentage of cleaved caspase 3-positive cells (Fig. 5F). In cells pretreated with VPA $(1 \mathrm{mM})$, the prosurvival effect of BDNF was almost completely abrogated. In serum-starved cells BDNF also significantly reduced the levels of PARP cleaved at Asp 186, and this effect was prevented by pre-exposure to VPA (Fig. 5G).

HDAC Inhibitors and HDAC1 Knockdown Mimic the Inhibitory Effect of VPA on TrkB Expression and Signaling. We investigated whether epigenetic mechanisms were involved in the downregulation of TrkB induced by VPA. To this goal, we first examined whether other HDAC inhibitors could mimic the effect of VPA. As shown in Supplemental Fig. 2, A-D, incubation of RA-differentiated SH-SY5Y cells with either VPA (1 mM); trichostatin A (300 nM), a broad spectrum HDAC inhibitor (Bolden et al., 2006); the class I inhibitor entinostat $(1 \mu \mathrm{M})$ (Saito et al., 1999; Hu et al., 2003); or MC 1568 (10 $\mu \mathrm{M}$ ), a class II HDAC inhibitor (Mai et al., 2005), elicited a time-dependent increase in the acetylation of histone $\mathrm{H} 3$ at Lys9/Lys14, which became significant 1-3 hours after drug exposure. Prolonged cell treatment with either romidepsin $(40 \mathrm{nM})$, a selective inhibitor of the class I members HDAC1 and 2, or the short-chain fatty acid sodium butyrate $(1 \mathrm{mM})$, which shares with VPA the ability to preferentially block class I and IIa HDAC (Bolden et al., 2006), markedly increased H3 acetylation (Supplemental Fig. 2E). Both HDAC6 and HDAC8 have been found to be expressed in human neuroblastoma cells, and their specific inhibition by tubacin and PCI-34051, respectively, has been shown to inhibit the growth and viability of these cells (Subramanian et al., 2011; Rettig et al., 2015). Consistent with previous studies (Haggarty et al., 2003; Balasubramanian et al., 2008), we found that both tubacin and PCI-34051, used at concentrations low enough to maintain their HDAC selectivity, failed to induce H3 histone acetylation (Supplemental Fig. 2E). A lack of effect was also observed in cells treated with the carboxamide derivative of VPA valpromide (1 mM) (Supplemental Fig. 2E), which lacks HDAC inhibitory activity (Phiel et al., 2001).

As observed with VPA, prolonged exposure (24 hours) of differentiated SH-SY5Y cells to either sodium butyrate (1 $\mathrm{mM})$, trichostatin $\mathrm{A}(300 \mathrm{nM})$, entinostat $(1 \mu \mathrm{M})$, or romidepsin (40 $\mathrm{nM}$ ) elicited a significant decrease in the expression of both TrkB-FL and TrkB-T isoforms (Fig. 6, A-C). Immunofluorescence assays indicated that entinostat $(1 \mu \mathrm{M})$ decreased the percentage of TrkB-positive cells by $80 \% \pm 4 \%(P<0.001, N=4)$ (Fig. 6D). Conversely, cell treatment with either MC1568 (10 $\mu \mathrm{M})$ (Fig. 6A), PCI-34051 (4 $\mu \mathrm{M})$, tubacin $(5 \mu \mathrm{M})$, or valpromide (1 mM) failed to affect TrkB expression (Fig. 6, E and F). Like VPA, trichostatin A and sodium butyrate induced a significant inhibition of BDNF-induced Akt phosphorylation, whereas valpromide and MC 1568 were without effect (Fig. 6, G and H). Entinostat $(0.5$ and $1 \mu \mathrm{M})$ caused a concentration-dependent inhibition of BDNF-stimulated phosphorylation of Akt and ERK1/2 (Fig. 6I). Similarly, cell treatment with romidepsin (20 and $40 \mathrm{nM})$ induced a significant decrease of BDNF-induced phosphorylation of Akt and GSK-3 $\beta$ (Fig. 6J).

We next examined the effects of knocking down HDAC1, an HDAC isoform that regulates neuronal survival and death (Bardai et al., 2012) and is inhibited by therapeutically relevant concentrations of VPA (Phiel et al., 2001). As shown in Fig. 6K, treatment of differentiated SH-SY5Y cells with siRNA targeting HDAC1 decreased HDAC1 protein levels by $60 \% \pm 12 \%(P<$ $0.05, N=3$ ) and increased the level of acetylated histone $\mathrm{H} 3$ by $70 \% \pm 10 \%(P<0.001, N=3)$ compared with control siRNAtreated cells. In HDAC1-depleted cells there was a significant decrease in the expression of both TrkB-FL and TrkB-T (Fig. 6L).

VPA Upregulates RUNX3 Expression. To investigate the involvement of transcriptional mechanisms in VPA-induced downregulation of TrkB, we examined the effects of VPA on the transcription factor RUNX3, a suppressor of TrkB expression (Kramer et al., 2006; Inoue et al., 2007). As shown in Fig. 7, A and B, exposure of differentiated SH-SY5Y cells to VPA induced a time- and concentration-dependent increase in RUNX3 protein levels. These effects occurred within the same concentration range of VPA required to increase histone $\mathrm{H} 3$ acetylation (Fig. 7B) and were mirrored by similar changes in the steadystate nuclear content of the transcription factor (Fig. 7C). An increase of RUNX3 levels was also observed in Kelly and LAN-1 cells following exposure to either VPA $(1 \mathrm{mM})$ or entinostat (1 $\mu \mathrm{M}$ ) (Supplemental Fig. 3, A and B).

Involvement of RUNX3 in VPA-Induced TrkB Downregulation. To investigate whether the enhanced expression of RUNX3 participates in TrkB downregulation by VPA, differentiated SH-SY5Y cells were transfected with siRNA duplexes targeting RUNX3. This treatment attenuated the increase in RUNX3 protein levels elicited by VPA and significantly reduced the downregulation of TrkB expression induced by the drug (Fig. 7D).

In human neuroblastoma cells, RUNX3 gene transcription has been reported to be under inhibitory control by the histone methyltransferase EZH2, the catalytic subunit of PRC2 (Wang et al., 2012). Exposure of RA-treated SH-SY5Y cells to VPA $(1 \mathrm{mM})$ reduced EZH2 protein levels by $60 \% \pm 5 \%(P<0.001$, $N=4$ ) (Fig. 7E). Moreover, in these cells, TrkB downregulation induced by HDAC1 depletion was associated with a decrease of EZH2 and an increase of RUNX3 protein levels (Fig. 7F). A significant decrease of EZH2 was also observed in Kelly and LAN-1 cells following treatment with either VPA $(1 \mathrm{mM})$ or entinostat ( $1 \mu \mathrm{M})$ (Supplemental Fig. 3, C and D).

To further investigate the involvement of the EZH2/RUNX3 axis in the regulation of TrkB expression, we examined the effects of the cyclopentenyl analog of 3-deazaadenosine 
A

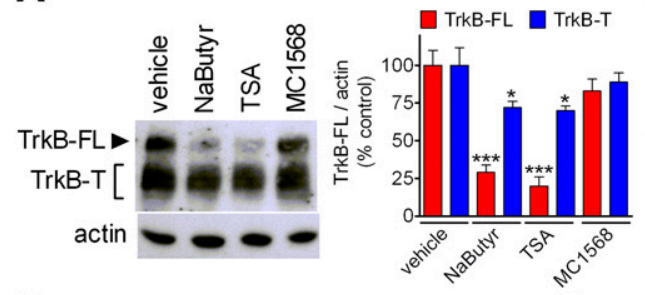

D

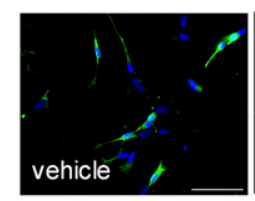

\begin{abstract}
E
\end{abstract}

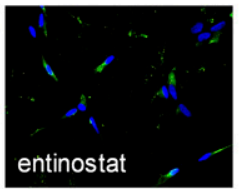

B

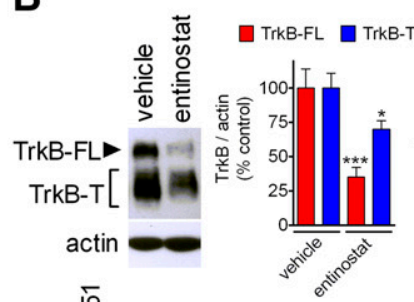

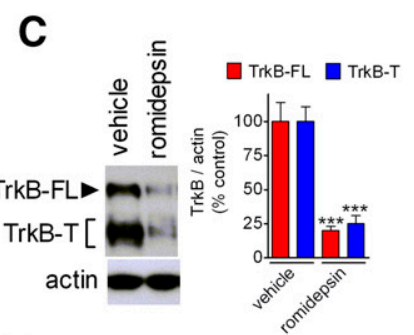

F

TrkB-FL $\square$ TrkB-T

TrkB-FL $>$

TrkB-T [

actin
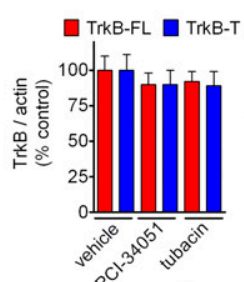

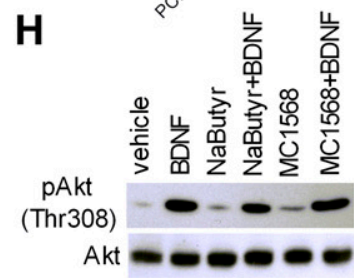

G
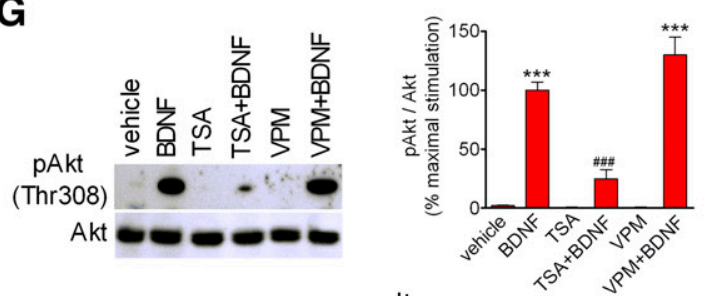

$\stackrel{\frac{0}{0}}{\frac{0}{\infty}} \sum_{\$}^{\infty}$

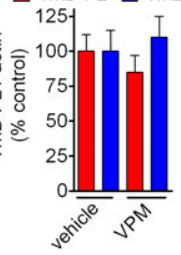

TrkB-FL -

TrkB-T $[\mathrm{e}$ )

$\operatorname{actin} 0$

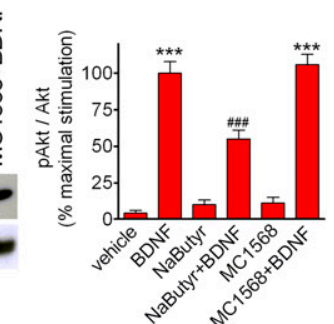

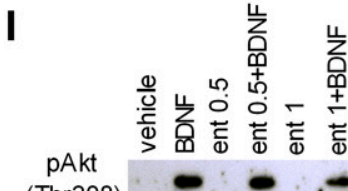

(Thr308)

Akt

pERK $1 / 2$

ERK $1 / 2=2=2=$

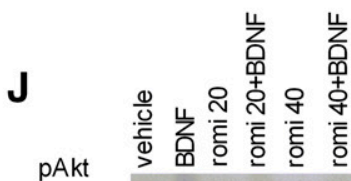

(Thr308)

Akt $=50=5$

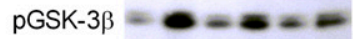

GSK-3 $\beta$

ol HDAC1

K
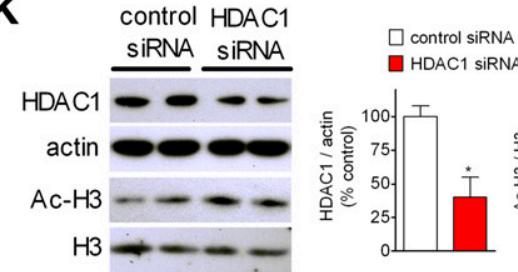
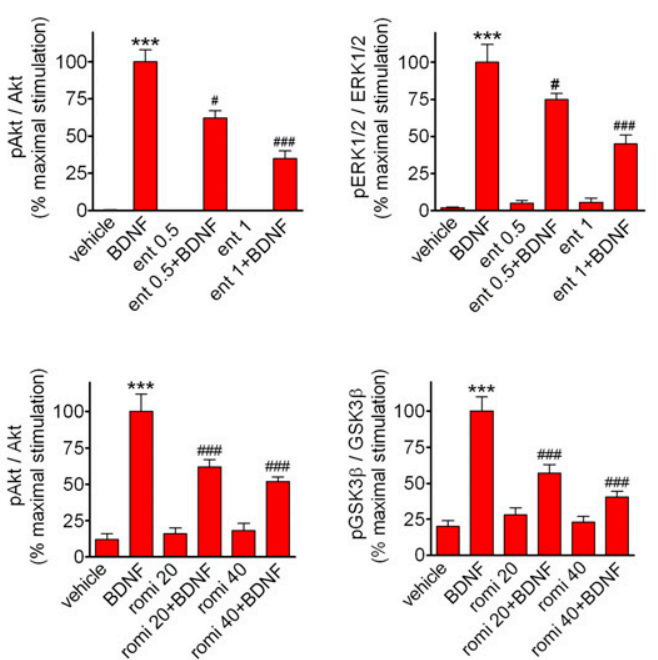
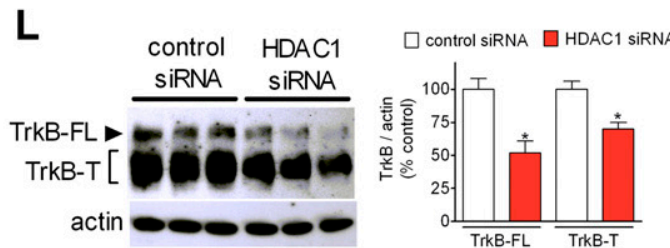

Fig. 6. HDAC inhibitors and HDAC1 knockdown mimic the inhibitory effect of VPA on TrkB expression and signaling. (A-C) SH-SY5Y cells were incubated for 24 hours with either vehicle, $1 \mathrm{mM}$ sodium butyrate, $300 \mathrm{nM}$ trichostatin A(TSA), $10 \mu \mathrm{M}$ MC1568 (A), $1 \mu \mathrm{M}$ entinostat (B), or $40 \mathrm{nM}$ romidepsin (C). Cell lysates were then analyzed for TrkB expression. Values are the mean \pm S.E.M. of four experiments. $* P<0.05 ; * * * P<0.001$ vs. the respective control (vehicle) by Student's $t$ test. (D) SH-SY5Y cells were incubated for 24 hours with either vehicle or $1 \mu \mathrm{M}$ entinostat and then TrkB expression was analyzed by immunofluorescence (green color) in nonpermeabilized cells stained with an antibody directed against an extracellular domain of the receptor. Nuclei were stained in blue with DAPI. Images are representative of four independent experiments. Scale bar, $25 \mu \mathrm{m}$. (E and F) SH-SY5Y cells were incubated for 24 hours with either vehicle, $4 \mu \mathrm{M}$ PCI-34051, $5 \mu \mathrm{M}$ tubacin (E), or $1 \mathrm{mM}$ valpromide (VPM) (F). Values are the mean \pm S.E.M. of four experiments. (G and H) SH-SY5Y cells were preincubated for 24 hours with either vehicle, $300 \mathrm{nM}$ TSA, $1 \mathrm{mM}$ VPM (G), $1 \mathrm{mM}$ sodium butyrate, or $10 \mu \mathrm{M}$ MC1568 (H) and then exposed for 5 minutes to $1 \mathrm{nM}$ BDNF. Values are the mean \pm S.E.M. of three experiments. (I and J) SH-SY5Y cells were preincubated for 24 hours with either vehicle, $0.5 \mu \mathrm{M}$ entinostat (ent 0.5), $1 \mu \mathrm{M}$ entinostat (ent 1) (I), $20 \mathrm{nM}$ romidepsin (romi 20), or $40 \mathrm{nM}$ romidepsin (romi 40) (J) and then exposed for 5 minutes to $1 \mathrm{nM}$ BDNF. Values are the mean \pm S.E.M. of four experiments. ${ }^{* * *} P<0.001$ vs. control; ${ }^{\#} P<0.05$; ${ }^{\# \# \#} P<0.001 \mathrm{vs}$. BDNF + vehicle by ANOVA followed by Newman-Keuls post hoc test. (K and L) SH-SY5Y cells were transfected with either control siRNA or HDAC1 siRNA duplexes and cell lysates were analyzed for HDAC1, H3 acetyl-Lys9/Lys14 (Ac-H3), total H3 (K), and TrkB expression (L). Each lane was loaded with a sample of an independent transfection. Values are the mean \pm S.E.M. of three experiments. ${ }^{*} P<0.05$; ${ }^{* *} P<0.001$ vs. control siRNA by Student's $t$ test. 
A

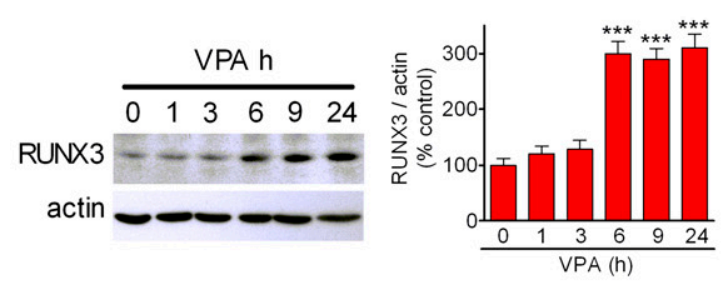

C

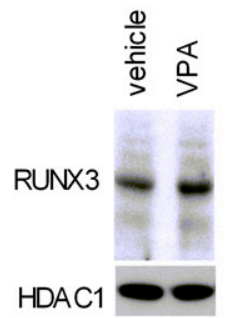

E
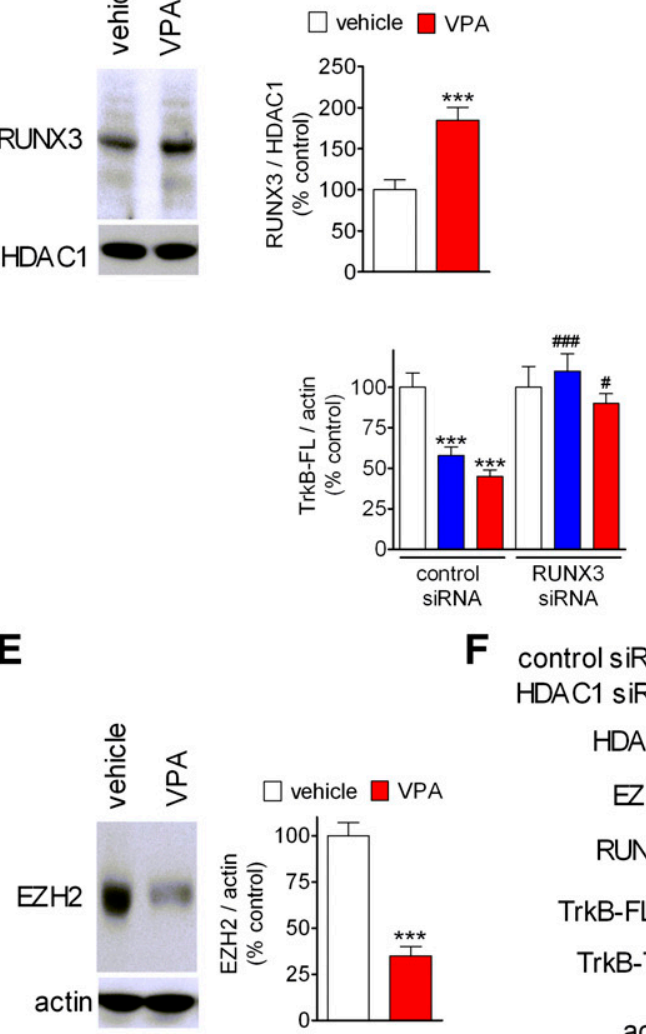

F control siRNA + -

B
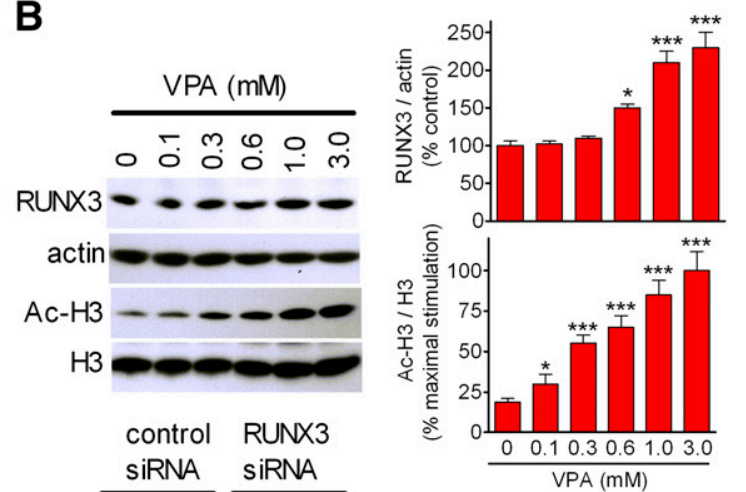

$\frac{\text { siRnA }}{0.0} \frac{\text { siRNA }}{0.0}$

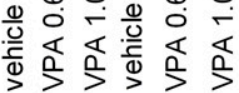

RUNX3

TrkB-FL

TrkB-T [

actin

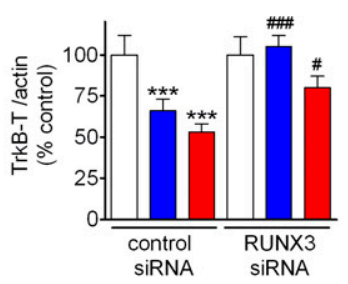

HDAC1 SIRNA - +
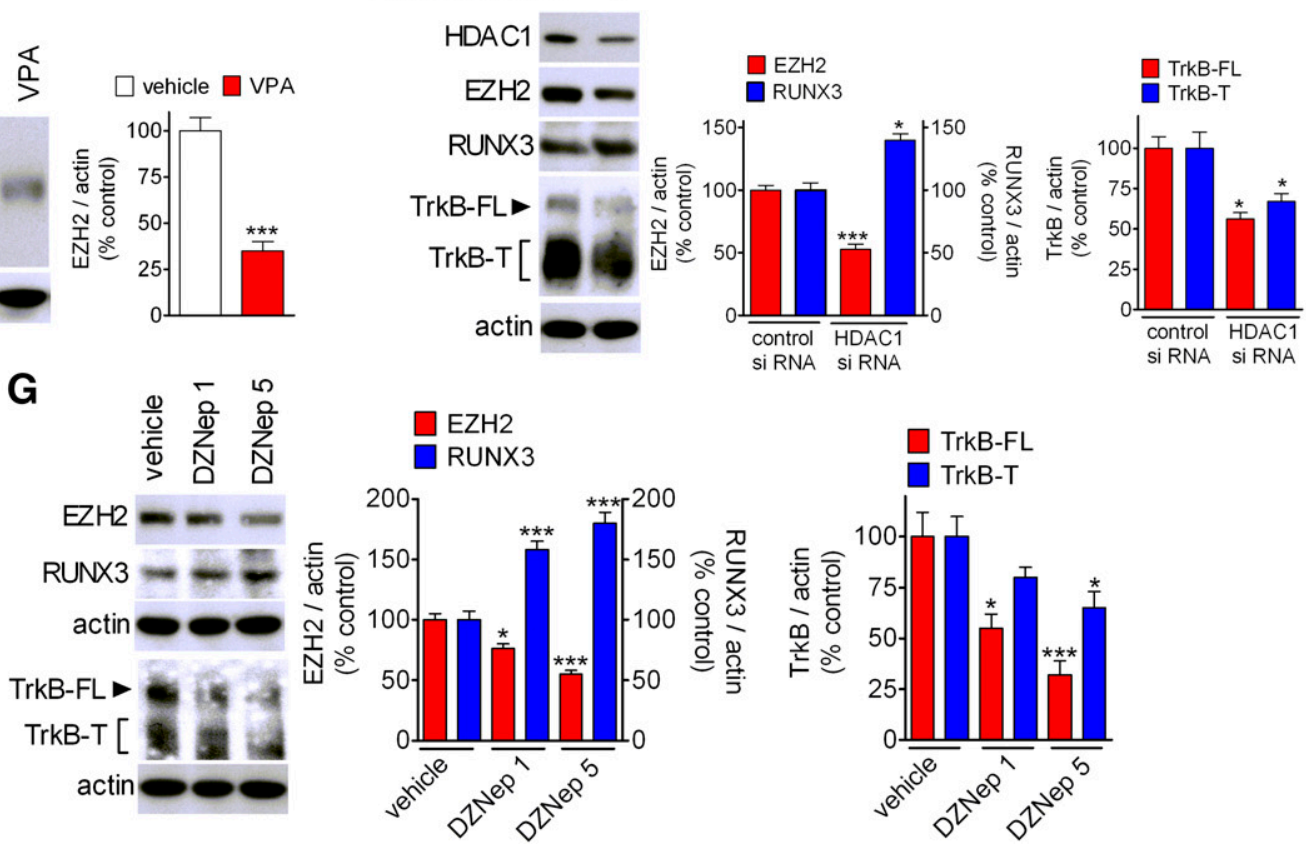

Fig. 7. Involvement of RUNX3 in VPA-induced downregulation of TrkB. (A) SH-SY5Y cells were incubated with $1 \mathrm{mM}$ VPA for the indicated periods of time. Zero time samples were treated with vehicle and used as control. (B) Cells were treated for 24 hours with the indicated concentrations of VPA. Cell lysates were analyzed for the protein levels of RUNX3 and H3-acetyl-Lys9/Lys14 (Ac-H3). Values are the mean \pm S.E.M. of four experiments. ${ }^{*} P<0.05$; *** $P<0.001$ vs. control (vehicle) by ANOVA followed by Newman-Keuls post hoc test. (C) SH-SY5Y cells were incubated with either vehicle or 1 mM VPA for 24 hours. Nuclear extracts were prepared and analyzed for RUNX3 and HDAC1 levels. Values are the mean \pm S.E.M. of four experiments. *** $P<0.001$ by Student's $t$ test. (D) SH-SY5Y cells transfected with either control siRNA or RUNX3 siRNA were incubated for 24 hours with either vehicle, $0.6 \mathrm{mM}$ VPA (VPA 0.6), or $1 \mathrm{mM}$ VPA (VPA 1.0). Cell lysates were then analyzed for RUNX3 and TrkB expression. Values are the mean \pm S.E.M. of three experiments. ${ }^{* * *} P<0.001$ vs. control siRNA + vehicle; ${ }^{\#} P<0.05$; ${ }^{\# \# \#} P<0.001$ vs. the corresponding value in control siRNA-treated group by Student's $t$ test. (E) SH-SY5Y cells were treated for 24 hours with either vehicle or $1 \mathrm{mM}$ VPA. Cell lysates were analyzed for EZH2 protein levels. Values 
DZNep, an inhibitor of $S$-adenosylhomocysteine hydrolase that has been shown to deplete the cellular content of PRC2 proteins in different cell types, including human neuroblastoma cells (Varambally et al., 2002; Tan et al., 2007; Wang et al., 2012). Incubation of RA-treated SH-SY5Y cells with DZNep (1 and $5 \mu \mathrm{M})$ reduced EZH2 and increased RUNX3 protein levels in a concentration-dependent manner (Fig. 7G). The epigenetic changes induced by DZNep were associated with a significant suppression of TrkB expression (Fig. 7G).

VPA Downregulates TrkB Expression and BDNF Signaling in Primary Mouse Cortical Neurons. To assess whether TrkB expression and function was altered by VPA in a nontumoral neuronal cell model, we employed primary cultures of mouse cortical neurons. As shown in Fig. 8A, prolonged exposure (24 hours) of these cultures to either VPA $(1 \mathrm{mM})$ or entinostat $(1 \mu \mathrm{M})$ produced a significant decrease in the levels of TrkB-FL and TrkB-T. The treatment with VPA and entinostat also induced a reduced receptor autophosphorylation and Akt activation in response to BDNF (1 nM) (Fig. 8, B and C). Moreover, as observed in human neuroblastoma cells, VPA $(1 \mathrm{mM})$ and entinostat $(1 \mu \mathrm{M})$ significantly increased histone $\mathrm{H} 3$ acetylation, decreased EZH2 levels, and increased RUNX3 expression (Fig. 8, D-F).

\section{Discussion}

By inhibiting HDAC activity and promoting chromatin remodeling, VPA may affect the expression of a variety of target genes, thus extending its spectrum of action beyond the acute regulation of neurotransmission. The present study shows that in neuronal cells, prolonged in vitro exposure to VPA and other HDAC inhibitors downregulates the expression and functional activity of TrkB, a key mediator of BDNF and neurotrophin 4 actions on neuronal growth, survival, and differentiation.

The downregulation of TrkB occurred at VPA concentrations $(0.6-1.0 \mathrm{mM})$ that are within the plasma concentrations associated with therapeutic effects in epilepsy (40-100 $\mu \mathrm{g} / \mathrm{ml}$, $\sim 0.3-0.7 \mathrm{mM}$ ) (Loscher, 2002). In clinical studies investigating the antitumor efficacy of VPA, the median total and free concentrations in plasma were 1.51 and $0.61 \mathrm{mM}$, respectively (Munster et al., 2009).

VPA-induced TrkB downregulation was associated with a marked decrease in the cell surface levels of the receptor. This change is relevant because previous studies have shown that in neurons the majority of TrkB immunoreactivity is located intracellularly, whereas the cell surface receptor population is the one responsive to neurotrophins (Du et al., 2000). It was therefore important to assess whether the downregulation of cell surface TrkB induced by VPA translated into a reduction in intracellular signaling and neurotrophic responses elicited by BDNF. We found that the exposure to VPA inhibited the activation of different components of the intracellular signaling cascade triggered by BDNF via TrkB-FL, such as PLC $\gamma 1$, Akt, GSK- $3 \beta$, and ERK1/2. Activation of Akt and the downstream suppression of GSK-3 $\beta$ through phosphorylation at Ser9 are critical events for promoting cell survival (Beurel and Jope,
2006), and their inhibition by VPA correlates with the observed drug-induced impairment of BDNF protection against apoptosis triggered by serum withdrawal. Similarly, the inhibition of ERK1/2 activation may underlie the VPA ability to counteract the changes in neuronal morphology induced by BDNF, as ERK1/2 has been shown to be required for neuronal differentiation and stimulation of neurite outgrowth elicited by the neurotrophin (Encinas et al., 1999; Huang and Reichardt, 2003). Collectively, these results suggest that the VPA-induced downregulation of TrkB-FL implicates relevant consequences with regard to the efficacy of BDNF in stimulating intracellular signaling and functional responses.

TrkB-T isoforms have been proposed to modulate BDNF signaling via TrkB-FL either by sequestering the neurotrophin (Biffo et al., 1995) and/or by acting as dominant negative (Eide et al., 1996). It has also been shown that TrkB-T may regulate the cell surface expression of TrkB-FL (Haapasalo et al., 2002). On the other hand, there is evidence that TrkB-T may signal independently of TrkB-FL and during development of mammalian cerebral cortex may mediate BDNF-induced differentiation of neural stem cells toward glial progenitors and astrocytes (Cheng et al., 2007). The observation that the exposure to VPA reduces the expression of TrkB-T suggests the possibility that the drug may also impair BDNF signaling through these receptors. Additional experiments in cells that predominantly express TrkB-T, such as glia cells (Rose et al., 2003), are required specifically to address this point.

A line of evidence supports the possibility that VPA downregulates TrkB through an epigenetic mechanism involving HDAC inhibition, depletion of EZH2, and upregulation of the TrkB suppressor RUNX3. Thus, like VPA different HDAC inhibitors downregulated the expression of TrkB and curtailed BDNF-induced intracellular signaling. These effects were induced not only by sodium butyrate and trichostatin A, which, like VPA, are known to inhibit multiple HDAC isoforms, but also by the class I inhibitors romidepsin and entinostat. This finding, together with the observation that the class II inhibitor MC 1568 was without effect, suggests the involvement of class I HDACs in the negative control of TrkB expression. This possibility is also supported by the observation that cell depletion of HDAC1 by siRNA treatment mimicked the inhibitory effect of VPA on TrkB expression.

HDAC1 and HDAC2 have been shown to interact with EZH2 through the EED protein, another PRC2 core component (van der Vlag and Otte, 1999; Varambally et al., 2002), and inhibition of HDAC or EZH2 activity has been shown to induce a pharmacologic disruption of the PRC2 complex likely by promoting the proteosome-mediated degradation of its core components (Varambally et al., 2002; Fiskus et al., 2006; Tan et al., 2007; Wang et al., 2012). In agreement with these data, we found that in human neuroblastoma cell lines and mouse cortical neurons, VPA and entinostat decreased EZH2 protein levels. Moreover, in differentiated SH-SY5Y cells, a reduced EZH2 expression was also found to be associated with TrkB downregulation induced by either HDAC1 knockdown or DZNep treatment.

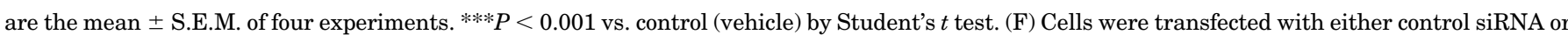

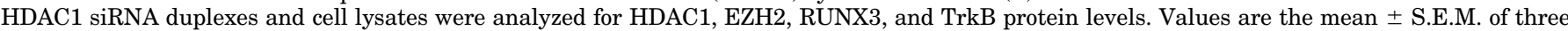

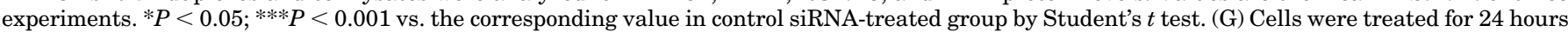

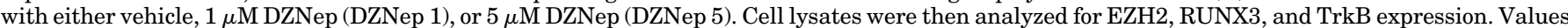
are the mean \pm S.E.M. of four experiments. ${ }^{*} P<0.05 ; * * * P<0.001$ vs. control (vehicle) by Student's $t$ test. 
A

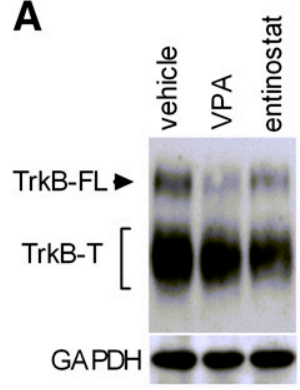

B

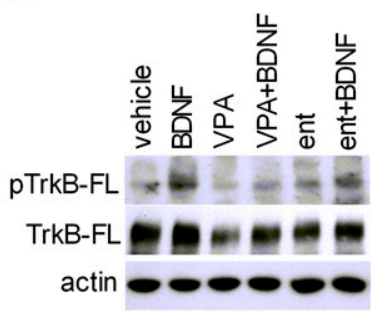

C

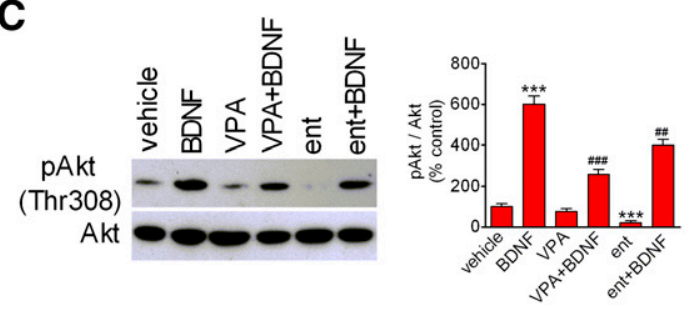

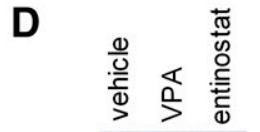

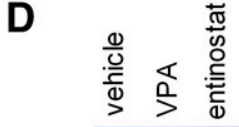

Ac-H3

H3

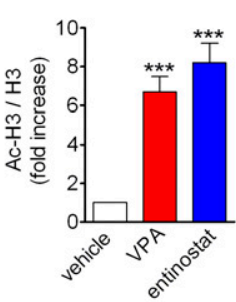

E

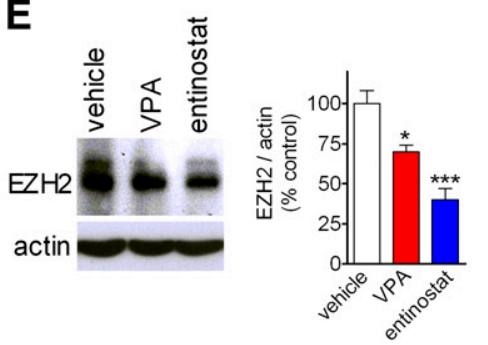

$\mathbf{F}$

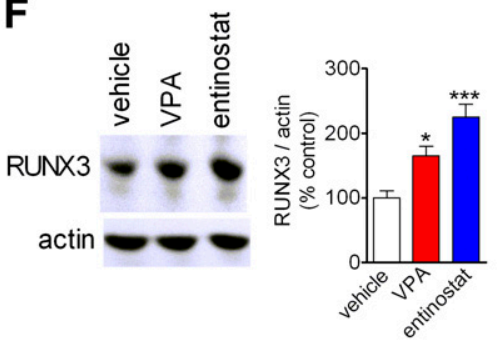

Fig. 8. VPA and entinostat downregulate TrkB expression and signaling in mouse primary cortical neurons. (A) Neuronal cultures were treated with either vehicle, $1 \mathrm{mM}$ VPA, or $1 \mu \mathrm{M}$ entinostat for 24 hours, and cell extracts were analyzed for the expression of TrkB isoforms. Values are the mean \pm S.E.M. of three experiments. $* P<0.05 ; * * * P<0.001$ vs. control (vehicle) by Student's $t$ test. (B and C) neurons were preincubated for 24 hours with either vehicle, $1 \mathrm{mM}$ VPA, or $1 \mu \mathrm{M}$ entinostat (ent) and then exposed for 5 minutes to either vehicle or $1 \mathrm{nM}$ BDNF. Cell extracts were analyzed for phospho-Tyr706/707-TrkB (pTrkB), TrkB-FL, phospho-Akt (Thr308), and total Akt. Values are the mean \pm S.E.M. of three experiments. *** $P<0.001$ vs. control (vehicle); ${ }^{\# \#} P<0.01$; ${ }^{\# \# \# ~} P<0.001$ vs. BDNF + vehicle by ANOVA followed by Newman-Keuls post hoc test. (D-F) Neurons were treated as indicated in (A) and cell extracts were then analyzed for Ac-H3, total H3, EZH2, and RUNX3 levels. Values are the mean \pm S.E.M. of three separate experiments. $* P<0.05 ; * * * P<0.001$ vs. control (vehicle) by Student's $t$ test.

EZH2 has been shown to participate in the epigenetic silencing of RUNX3 gene (Chuang and Ito, 2010) and in human neuroblastoma cells EZH2 depletion has been found to derepress RUNX3 expression (Wang et al., 2012). RUNX3 has been shown to suppress TrkB in differentiating sensory neurons (Kramer et al., 2006), and, when transfected in RA-treated neuroblastoma cells, to abrogate TrkB expression likely by direct binding to distal regulatory sequences in the TrkB gene (Inoue et al., 2007). Consistent with their ability to decrease EZH2 levels, VPA, entinostat, HDAC1 knockdown, and DZNep increased the cellular content of RUNX3. Moreover, silencing of RUNX3 gene prevented VPA-induced RUNX3 elevation and TrkB downregulation. The possibility that VPA downregulates TrkB by promoting the inhibition of TrkB gene expression through RUNX3 is also consistent with the finding that the drug caused a significant decrease in TrkB mRNA levels.

Whether VPA can regulate TrkB also through non-HDACdependent processes remains to be investigated. VPA has been shown to regulate intracellular pathways, such as the MEK/ ERK and the PI3K/Akt/GSK-3 $\beta$ cascades, which are known to regulate gene transcription (Chiu et al., 2013). Importantly, these pathways commonly lead to activation of the transcription factor CREB, which has been shown to act as a positive regulator of the TrkB gene in neurons (Lei and Parada, 2007). Thus, the outcome of this process would likely be upregulation, rather than downregulation, of TrkB.

Prolonged exposure to IFN- $\beta$ has been reported to downregulate TrkB expression and functional activity in differentiated SH-SY5Y cells and primary neurons (Dedoni et al., 2012). A rapid and marked decrease of cell surface expression of TrkB also occurs following treatment of neurons with BDNF (Sommerfeld et al., 2000). In the present study, we did not examine the effects of these TrkB downregulators under the same experimental conditions used for VPA, so that the relative efficacies of these different agents in suppressing TrkB were not determined.

The finding that VPA and other HDAC inhibitors downregulate TrkB expression and signaling may have important implications for the therapeutic use of these compounds, notably as antitumor agents. TrkB is highly expressed in neuroblastoma and in aggressive tumors of pancreas, prostate, and lymphoid tissue. In human neuroblastoma, the occurrence of BDNF/ TrkB signaling is known to promote tumor survival, resistance to chemotherapy, anoikis, and metastasis (Nakagawara et al., 1994; Matsumoto et al., 1995; Middlemas et al., 1999; Douma et al., 2004). In neuroblastoma specimens, TrkB-FL expression has been found to be associated with MYCN gene amplification, 
a negative prognostic marker of this tumor (Nakagawara et al., 1994). Preclinical studies have demonstrated that HDAC inhibitors possess potent anticancer activity for highly malignant tumors, such as neuroblastoma (Witt et al., 2009; West and Johnstone, 2014). The present study suggests that VPA and class I HDAC inhibitors may be particularly useful for treating aggressive tumors expressing TrkB.

In differentiated SH-SY5Y cells, the expression of TrkA was not significantly affected by VPA, whereas the protein levels of TrkC and p75NTR were markedly elevated by the drug. This indicates that VPA treatment differentially affected neurotrophin receptor expression. Interestingly, the expression of TrkC is a favorable prognostic factor in neuroblastomas (Yamashiro et al., 1996; Ryden et al., 1996) and medulloblastomas (Segal et al., 1994). Unlike TrkA and TrkB, both p75NTR and TrkC have been shown to act as dependence receptors, which in the absence of the ligand trigger apoptotic cell death (Bouzas-Rodriguez et al., 2010). Thus, their induction by VPA may constitute an additional mechanism supporting the antitumor action of this drug.

We observed that VPA and entinostat downregulated TrkB expression and signaling also in mouse primary cortical neurons and altered EZH2 and RUNX3 protein levels in a manner similar to that observed in human neuroblastoma cells. These results suggest that the drugs are able to affect the epigenetic control of TrkB expression also in nontumor neuronal cells and independently of cell pretreatment with RA. The functional significance of VPA-induced TrkB downregulation in cortical neurons remains to be established. TrkB inhibition is considered a potential therapeutic target for some central nervous systemrelated disorders, such as epilepsy and neuropathic pain, which are effectively treated with VPA (Boulle et al., 2012). Additional studies are required to investigate whether the downregulation of TrkB contributes to the beneficial effects of VPA in these neurologic diseases.

\section{Authorship Contributions}

Participated in research design: Dedoni, Olianas, Ingianni, Onali. Conducted experiments: Dedoni, Marras, Olianas.

Performed data analysis: Dedoni, Marras, Olianas.

Wrote or contributed to the writing of the manuscript: Dedoni, Olianas, Ingianni, Onali.

\section{References}

Autry AE and Monteggia LM (2012) Brain-derived neurotrophic factor and neuropsychiatric disorders. Pharmacol Rev 64:238-258.

Balasubramanian S, Ramos J, Luo W, Sirisawad M, Verner E, and Buggy JJ (2008) A novel histone deacetylase 8 (HDAC8)-specific inhibitor PCI-34051 induces apoptosis in T-cell lymphomas. Leukemia 22:1026-1034.

Bardai FH, Price V, Zaayman M, Wang L, and D'Mello SR (2012) Histone deacetylase-1 (HDAC1) is a molecular switch between neuronal survival and death. J Biol Chem 287:35444-35453.

Beurel E and Jope RS (2006) The paradoxical pro- and anti-apoptotic actions of GSK3 in the intrinsic and extrinsic apoptosis signaling pathways. Prog Neurobiol 79 173-189.

Biffo S, Offenhäuser N, Carter BD, and Barde YA (1995) Selective binding and internalisation by truncated receptors restrict the availability of BDNF during development. Development 121:2461-2470.

Bolden JE, Peart MJ, and Johnstone RW (2006) Anticancer activities of histone deacetylase inhibitors. Nat Rev Drug Discov 5:769-784.

Boulle F, Kenis G, Cazorla M, Hamon M, Steinbusch HWM, Lanfumey L, and van den Hove DLA (2012) TrkB inhibition as a therapeutic target for CNS-related disorders. Prog Neurobiol 98:197-206.

Bouzas-Rodriguez J, Cabrera JR, Delloye-Bourgeois C, Ichim G, Delcros J-G, Raquin M-A, Rousseau R, Combaret V, Bénard J, Tauszig-Delamasure S, et al. (2010) Neurotrophin-3 production promotes human neuroblastoma cell survival by inhibiting TrkC-induced apoptosis. J Clin Invest 120:850-858.

Cesca F, Baldelli P, Valtorta F, and Benfenati F (2010) The synapsins: key actors of synapse function and plasticity. Prog Neurobiol 91:313-348.

Chao MV (2003) Neurotrophins and their receptors: a convergence point for many signalling pathways. Nat Rev Neurosci 4:299-309.
Chateauvieux S, Morceau F, Dicato M, and Diederich M (2010) Molecular and therapeutic potential and toxicity of valproic acid. $J$ Biomed Biotechnol 2010: 479364.

Cheng A, Coksaygan T, Tang H, Khatri R, Balice-Gordon RJ, Rao MS, and Mattson MP (2007) Truncated tyrosine kinase B brain-derived neurotrophic factor receptor directs cortical neural stem cells to a glial cell fate by a novel signaling mechanism. $J$ Neurochem 100:1515-1530.

Chiu C-T, Wang Z, Hunsberger JG, and Chuang D-M (2013) Therapeutic potential of mood stabilizers lithium and valproic acid: beyond bipolar disorder. Pharmacol Rev 65:105-142.

Chuang LSH and Ito Y (2010) RUNX3 is multifunctional in carcinogenesis of multiple solid tumors. Oncogene 29:2605-2615.

Dedoni S, Olianas MC, Ingianni A, and Onali P (2012) Type I interferons impair BDNF-induced cell signaling and neurotrophic activity in differentiated human SH-SY5Y neuroblastoma cells and mouse primary cortical neurons. J Neurochem 122:58-71.

Dedoni S, Olianas MC, Ingianni A, and Onali P (2017) Interferon- $\beta$ inhibits neurotrophin 3 signalling and pro-survival activity by upregulating the expression of truncated TrkC-T1 receptor. Mol Neurobiol 54:1825-1843.

Dedoni S, Olianas MC, and Onali P (2010) Interferon- $\beta$ induces apoptosis in human SH-SY5Y neuroblastoma cells through activation of JAK-STAT signaling and downregulation of PI3K/Akt pathway. J Neurochem 115:1421-1433.

Douma S, Van Laar T, Zevenhoven J, Meuwissen R, Van Garderen E, and Peeper DS (2004) Suppression of anoikis and induction of metastasis by the neurotrophic receptor TrkB. Nature 430:1034-1039.

Du J, Feng L, Yang F, and Lu B (2000) Activity- and $\mathrm{Ca}^{2+}{ }^{2+}$ )-dependent modulation of surface expression of brain-derived neurotrophic factor receptors in hippocampal neurons. J Cell Biol 150:1423-1434.

Eide FF, Vining ER, Eide BL, Zang K, Wang XY, and Reichardt LF (1996) Naturally occurring truncated trkB receptors have dominant inhibitory effects on brainderived neurotrophic factor signaling. J Neurosci 16:3123-3129.

Encinas M, Iglesias M, Llecha N, and Comella JX (1999) Extracellular-regulated kinases and phosphatidylinositol 3-kinase are involved in brain-derived neurotrophic factor-mediated survival and neuritogenesis of the neuroblastoma cell line SH-SY5Y. J Neurochem 73:1409-1421.

Fiskus W, Pranpat M, Balasis M, Herger B, Rao R, Chinnaiyan A, Atadja P, and Bhalla K (2006) Histone deacetylase inhibitors deplete enhancer of zeste 2 and associated polycomb repressive complex 2 proteins in human acute leukemia cells. Mol Cancer Ther 5:3096-3104.

Fujii S, Ito K, Ito Y, and Ochiai A (2008) Enhancer of zeste homologue 2 (EZH2) down-regulates RUNX3 by increasing histone H3 methylation. J Biol Chem 283: 17324-17332.

Fukuchi M, Nii T, Ishimaru N, Minamino A, Hara D, Takasaki I, Tabuchi A, and Tsuda M (2009) Valproic acid induces up- or down-regulation of gene expression responsible for the neuronal excitation and inhibition in rat cortical neurons through its epigenetic actions. Neurosci Res 65:35-43.

Garner CC and Matus A (1988) Different forms of microtubule-associated protein 2 are encoded by separate mRNA transcripts. J Cell Biol 106:779-783.

Göttlicher M, Minucci S, Zhu P, Krämer OH, Schimpf A, Giavara S, Sleeman JP, Lo Coco F, Nervi C, Pelicci PG, et al. (2001) Valproic acid defines a novel class of HDAC inhibitors inducing differentiation of transformed cells. EMBO J 20:6969-6978.

Haapasalo A, Sipola I, Larsson K, Akerman KEO, Stoilov P, Stamm S, Wong G, and Castren E (2002) Regulation of TRKB surface expression by brainderived neurotrophic factor and truncated TRKB isoforms. J Biol Chem $\mathbf{2 7 7}$ $43160-43167$

Haggarty SJ, Koeller KM, Wong JC, Grozinger CM, and Schreiber SL (2003) Domainselective small-molecule inhibitor of histone deacetylase 6 (HDAC6)-mediated tubulin deacetylation. Proc Natl Acad Sci USA 100:4389-4394.

Hu E, Dul E, Sung C-M, Chen Z, Kirkpatrick R, Zhang G-F, Johanson K, Liu R, Lago A, Hofmann G, et al. (2003) Identification of novel isoform-selective inhibitors within class I histone deacetylases. J Pharmacol Exp Ther 307:720-728.

Huang EJ and Reichardt LF (2003) Trk receptors: roles in neuronal signal transduction. Annu Rev Biochem 72:609-642.

Inoue K, Ito K, Osato M, Lee B, Bae S-C, and Ito Y (2007) The transcription factor Runx3 represses the neurotrophin receptor TrkB during lineage commitment of dorsal root ganglion neurons. J Biol Chem 282:24175-24184.

Kaplan DR, Matsumoto K, Lucarelli E, and Thiele CJ; Eukaryotic Signal Transduction Group (1993) Induction of TrkB by retinoic acid mediates biologic responsiveness to BDNF and differentiation of human neuroblastoma cells. Neuron 11:321-331.

Kim HK, Kim JW, Zilberstein A, Margolis B, Kim JG, Schlessinger J, and Rhee SG (1991) PDGF stimulation of inositol phospholipid hydrolysis requires PLC-gamma 1 phosphorylation on tyrosine residues 783 and 1254. Cell 65:435-441.

Klein R, Conway D, Parada LF, and Barbacid M (1990) The trkB tyrosine protein kinase gene codes for a second neurogenic receptor that lacks the catalytic kinase domain. Cell 61:647-656.

Kramer I, Sigrist M, de Nooij JC, Taniuchi I, Jessell TM, and Arber S (2006) A role for Runx transcription factor signaling in dorsal root ganglion sensory neuron diversification. Neuron 49:379-393.

Lei L and Parada LF (2007) Transcriptional regulation of Trk family neurotrophin receptors. Cell Mol Life Sci 64:522-532.

Löscher W (2002) Basic pharmacology of valproate: a review after 35 years of clinical use for the treatment of epilepsy. CNS Drugs 16:669-694.

Macleod MR, Allsopp TE, McLuckie J, and Kelly JS (2001) Serum withdrawal causes apoptosis in SHSY 5Y cells. Brain Res 889:308-315.

Mai A, Massa S, Pezzi R, Simeoni S, Rotili D, Nebbioso A, Scognamiglio A, Altucci L, Loidl P, and Brosch G (2005) Class II (IIa)-selective histone deacetylase inhibitors. 1. Synthesis and biological evaluation of novel (aryloxopropenyl)pyrrolyl hydroxyamides. J Med Chem 48:3344-3353. 
Matsumoto K, Wada RK, Yamashiro JM, Kaplan DR, and Thiele CJ (1995) Expression of brain-derived neurotrophic factor and p145TrkB affects survival, differentiation, and invasiveness of human neuroblastoma cells. Cancer Res 55:1798-1806.

McAllister AK, Katz LC, and Lo DC (1999) Neurotrophins and synaptic plasticity. Annu Rev Neurosci 22:295-318.

Middlemas DS, Kihl BK, Zhou J, and Zhu X (1999) Brain-derived neurotrophic factor promotes survival and chemoprotection of human neuroblastoma cells. $J$ Biol Chem 274:16451-16460.

Munster P, Marchion D, Bicaku E, Lacevic M, Kim J, Centeno B, Daud A, Neuger A, Minton S, and Sullivan D (2009) Clinical and biological effects of valproic acid as a histone deacetylase inhibitor on tumor and surrogate tissues: phase I/II trial of valproic acid and epirubicin/FEC. Clin Cancer Res 15:2488-2496.

Nakagawara A, Azar CG, Scavarda NJ, and Brodeur GM (1994) Expression and function of TRK-B and BDNF in human neuroblastomas. Mol Cell Biol 14:759-767.

Nalivaeva NN, Belyaev ND, and Turner AJ (2009) Sodium valproate: an old drug with new roles. Trends Pharmacol Sci 30:509-514.

Park H and Poo MM (2013) Neurotrophin regulation of neural circuit development and function. Nat Rev Neurosci 14:7-23.

Phiel CJ, Zhang F, Huang EY, Guenther MG, Lazar MA, and Klein PS (2001) Histone deacetylase is a direct target of valproic acid, a potent anticonvulsant, mood stabilizer, and teratogen. $J$ Biol Chem 276:36734-36741.

Rettig I, Koeneke E, Trippel F, Mueller WC, Burhenne J, Kopp-Schneider A, Fabian J, Schober A, Fernekorn U, von Deimling A, et al. (2015) Selective inhibition of HDAC8 decreases neuroblastoma growth in vitro and in vivo and enhances retinoic acidmediated differentiation. Cell Death Dis 6:e1657.

Rose CR, Blum R, Pichler B, Lepier A, Kafitz KW, and Konnerth A (2003) Truncated TrkB-T1 mediates neurotrophin-evoked calcium signalling in glia cells. Nature 426:74-78.

Rydén M, Sehgal R, Dominici C, Schilling FH, Ibáñez CF, and Kogner P (1996) Expression of mRNA for the neurotrophin receptor trkC in neuroblastomas with favourable tumour stage and good prognosis. Br J Cancer 74:773-779.

Saito A, Yamashita T, Mariko Y, Nosaka Y, Tsuchiya K, Ando T, Suzuki T, Tsuruo T, and Nakanishi O (1999) A synthetic inhibitor of histone deacetylase, MS-27-275 with marked in vivo antitumor activity against human tumors. Proc Natl Acad Sci USA 96:4592-4597.

Segal RA, Goumnerova LC, Kwon YK, Stiles CD, and Pomeroy SL (1994) Expression of the neurotrophin receptor TrkC is linked to a favorable outcome in medulloblastoma. Proc Natl Acad Sci USA 91:12867-12871.

Sommerfeld MT, Schweigreiter R, Barde Y-A, and Hoppe E (2000) Down-regulation of the neurotrophin receptor TrkB following ligand binding. Evidence for an involvement of the proteasome and differential regulation of TrkA and TrkB. J Biol Chem 275:8982-8990.

Stoilov P, Castren E, and Stamm S (2002) Analysis of the human TrkB gene genomic organization reveals novel TrkB isoforms, unusual gene length, and splicing mechanism. Biochem Biophys Res Commun 290:1054-1065.

Subramanian C, Jarzembowski JA, Opipari AW Jr, Castle VP, and Kwok RPS (2011) HDAC6 deacetylates Ku70 and regulates Ku70-Bax binding in neuroblastoma. Neoplasia 13:726-734

Tan J, Yang X, Zhuang L, Jiang X, Chen W, Lee PL, Karuturi RKM, Tan PBO, Liu ET, and Yu Q (2007) Pharmacologic disruption of Polycomb-repressive complex 2mediated gene repression selectively induces apoptosis in cancer cells. Genes Dev 21:1050-1063.

Thiagalingam S, Cheng K-H, Lee HJ, Mineva N, Thiagalingam A, and Ponte JF (2003) Histone deacetylases: unique players in shaping the epigenetic histone code. Ann N Y Acad Sci 983:84-100.

van der Vlag J and Otte AP (1999) Transcriptional repression mediated by the human polycomb-group protein EED involves histone deacetylation. Nat Genet 23: 474-478.

Varambally S, Dhanasekaran SM, Zhou M, Barrette TR, Kumar-Sinha C, Sanda MG, Ghosh D, Pienta KJ, Sewalt RGAB, Otte AP, et al. (2002) The polycomb group protein EZH2 is involved in progression of prostate cancer. Nature 419 : 624-629.

Wang C, Liu Z, Woo C-W, Li Z, Wang L, Wei JS, Marquez VE, Bates SE, Jin Q, Khan J, et al. (2012) EZH2 Mediates epigenetic silencing of neuroblastoma suppressor genes CASZ1, CLU, RUNX3, and NGFR. Cancer Res 72:315-324.

West AC and Johnstone RW (2014) New and emerging HDAC inhibitors for cancer treatment. J Clin Invest 124:30-39.

Witt O, Deubzer HE, Lodrini M, Milde T, and Oehme I (2009) Targeting histone deacetylases in neuroblastoma. Curr Pharm Des 15:436-447.

Yamashiro DJ, Nakagawara A, Ikegaki N, Liu XG, and Brodeur GM (1996) Expression of TrkC in favorable human neuroblastomas. Oncogene 12:37-41.

Yasuda S, Liang MH, Marinova Z, Yahyavi A, and Chuang D-M (2009) The mood stabilizers lithium and valproate selectively activate the promoter IV of brainderived neurotrophic factor in neurons. Mol Psychiatry 14:51-59.

Address correspondence to: Pierluigi Onali, Department of Biomedical Sciences, Section of Neurosciences, Cittadella Universitaria di Monserrato, 09042 Monserrato (CA), Italy. E-mail: onali@unica.it 